\title{
The Navier-Stokes Equations with Particle Methods
}

\section{Werner Varnhorn}

\author{
Faculty of Mathematics, University of Kassel, Germany \\ varnhorn@mathematik.uni-kassel.de
}

\begin{abstract}
The non-stationary nonlinear Navier-Stokes equations describe the motion of a viscous incompressible fluid flow for $0<t \leqslant T$ in some bounded three-dimensional domain. Up to now it is not known wether these equations are well-posed or not. Therefore we use a particle method to develop a system of approximate equations. We show that this system can be solved uniquely and globally in time and that its solution has a high degree of spatial regularity. Moreover we prove that the system of approximate solutions has an accumulation point satisfying the Navier-Stokes equations in a weak sense.
\end{abstract}

Key-Words: Navier-Stokes approximation, weak solutions, compatibility condition Mathematics Subject Classification (2000): 35B65, 35D05, 76D05

\section{Introduction}

Let $T>0$ be given and $\Omega \subseteq \mathbb{R}^{3}$ be a bounded domain with a smooth compact boundary $\partial \Omega$. In $\Omega$ we consider a non-stationary viscous incompressible fluid flow and assume that it can be described by the Navier-Stokes equations

$$
\begin{aligned}
\partial_{t} v-\nu \Delta v+\nabla p+v \cdot \nabla v & =F, \\
\nabla \cdot v & =0, \\
v_{\mid \partial \Omega}=0, \quad v_{\mid t=0} & =v_{0} .
\end{aligned}
$$


These equations represent a system of nonlinear partial differential equations concerning four unknown functions: the velocity vector $v=\left(v_{1}(t, x), v_{2}(t, x), v_{3}(t, x)\right)$ and the (scalar) kinematic pressure function $p=p(t, x)$ of the fluid at the time $t \in(0, T)$ in the point $x \in \Omega$. The constant $\nu>0$ (kinematic viscosity), the external force density $F$, and the initial velocity $v_{0}$ are given data. In $\left(N_{0}\right) \partial_{t} v$ means the partial derivative with respect to the time $t, \Delta$ is the Laplace operator in $\mathbb{R}^{3}$, and $\nabla=\left(\partial_{1}, \partial_{2}, \partial_{3}\right)$ the gradient, where $\partial_{j}=\frac{\partial}{\partial x_{j}}$ denotes the partial derivative with respect to $x_{j}(j=1,2,3)$. From the physical point of view, the nonlinear convective term $v \cdot \nabla v$ is a result of the total derivative of the velocity field. Here the operator $v \cdot \nabla$ has to be applied to each component $v_{j}$ of $v$. In the fourth equation $\nabla \cdot v=\partial_{1} v_{1}+\partial_{2} v_{2}+\partial_{3} v_{3}$ defines the divergence of $v$, which vanishes due to the incompressibility of the fluid. Finally, the no-slip boundary condition $v_{\mid \partial \Omega}=0$ expresses that the fluid adheres to the boundary $\partial \Omega$.

Let us assume that smooth data are given without any smallness assumptions. Then the problem to construct a solution $v, \nabla p$ of $\left(N_{0}\right)$, which is uniquely determined and exists globally in time, has not been solved in the 3 -d case considered here (see for example [6], [7], $[8])$. Consequently, there is no globally stable approximation scheme for $\left(N_{0}\right)$ up to now.

In the present paper we use particle methods to approximate the Navier-Stokes equations by globally and uniquely solvable systems. To do so, let us consider, in particular, the nonlinear convective term $v \cdot \nabla v$, which is responsable for the non-global existence of the solution. From the physical point of view, this term results from the total (material) derivative of the velocity field $v$, and therefore the use of total differences in connection with particle methods seems to be reasonable. This leads to an approximation of the nonlinear term by some kind of central total difference quotient, which does not destroy the conservation of energy. The corresponding particle method and the properties of the trajectories are studied in Section 2 and Section 3. Using an additional time delay, the resulting system can be linearized. This requires a certain initial procedure to start, which is carried out in Section 4. Constructing sufficiently regular solutions even at initial time $t=0$, a non-local compatibility condition arises, not checkable for given data. This condition can be satisfied, however, by a construction of suitable initial velocities from a prescribed initial acceleration vanishing on the boundary $\partial \Omega$. In the following sections 5,6 , and 7 the approximate system is investigated with energy methods: A Galerkin ansatz based on the eigenfunctions of the Stokes operator $-P \Delta$ leads to a unique, for $0 \leq t \leq T$ globally existing, strongly $H_{4}$ continuous regular solution. In Section 8 we prove that the Navier Stokes equations $\left(N_{0}\right)$ can be re-obtained from this system in a certain sense, if the finite differences tend to zero: In this case there always exists a subsequence of the solution sequence with limit function $v$ such that $v$ is a weak solution of $\left(N_{0}\right)$. Finally, in Section 9 local convergence properties of the whole sequence to the locally in time existing strong solution on the Navier Stokes system $\left(N_{0}\right)$ are proved.

At this stage let us outline the notation: We use $\mathbb{N}:=\{1,2,3, \ldots\}, \mathbb{N}_{0}:=\mathbb{N} \cup\{0\}$, and denote by $\mathbb{R}$ the real numbers. 
For $x, y \in \mathbb{R}^{3}, x=\left(x_{1}, x_{2}, x_{3}\right), y=\left(y_{1}, y_{2}, y_{3}\right)$, let

$$
x \cdot y:=\sum_{i=1}^{3} x_{i} y_{i}
$$

be the scalar product of $x, y$ and $|x|:=\sqrt{x \cdot x}$ the Euclidian norm of $x$.

Throughout the paper, $\Omega$ denotes a bounded domain in $\mathbb{R}^{3}$ with smooth boundary $\partial \Omega$ at least of class $C^{2}$. We set $\bar{\Omega}:=\Omega \cup \partial \Omega$ and $\Omega^{c}:=\mathbb{R}^{3} \backslash \bar{\Omega}$. For $T>0$ let $\Omega_{T}:=(0, T) \times \Omega$. By $N(x)$ we mean the exterior unit normal with respect to $\Omega$ in $x \in \partial \Omega$.

We use the same symbols for scalar-valued and vector-valued functions. The partial derivative of some functions $v$ with respect to the $i$-th coordinate is denoted by $\partial_{i} v$, for a multi-index $\alpha \in \mathbb{N}_{0}^{3}$ let $\partial^{\alpha} v:=\partial_{1}^{\alpha_{1}} \partial_{2}^{\alpha_{2}} \partial_{3}^{\alpha_{3}} v$ and

$$
|\alpha|:=\sum_{i=1}^{3} \alpha_{i}
$$

the length of $\alpha$.

Setting $\nabla:=\left(\partial_{1}, \partial_{2}, \partial_{3}\right)$ we denote by

$$
\operatorname{div} v:=\nabla \cdot v:=\sum_{i=1}^{3} \partial_{i} v_{i}
$$

the divergence of the function $v=\left(v_{1}, v_{2}, v_{3}\right)$, and $\nabla v$ is the $3 \times 3$-matrix defined by $\left(\partial_{i} v_{j}\right)_{j i}$

For a domain $A \subseteq \mathbb{R}^{n}$ and $m \in \mathbb{N}_{0}$, let $C^{m}(A)$ be the space of functions being $m$-times continuously differentiable in $A$, and let $C^{m}(\bar{A})$ denote the subspace of functions, which together with all their derivatives up to and including order $m$ - can be extended continuously onto $\partial A$. We set

$$
C^{\infty}(A):=\bigcap_{m \in \mathbb{N}} C^{m}(A)
$$

and define $C_{0}^{\infty}(A)$ to be the subspace of $C^{\infty}(A)$ containing functions with a compact support in $A$. The subspace $C_{0, \sigma}^{\infty}(A)$ contains vector functions in $C_{0}^{\infty}(A)$, which are divergence free, in addition.

If $v:(t, x) \rightarrow v(t, x)$ is a function defined in $\Omega_{T}$ we denote by $v(t):=v(t, \cdot)$ the function defined by $x \rightarrow(v(t))(x):=v(t, x)$ in $\Omega, t \in(0, T)$. For $T_{1}, T_{2} \in \mathbb{R}, T_{1}<T_{2}$, and some Banach space $B$ let $C\left(\left[T_{2}, T_{2}\right], B\right)$ be the space of $B$-valued function being uniformly continuous in $\left[T_{1}, T_{2}\right]$.

By $L_{p}(\Omega)(1 \leq p<\infty)$ we denote the usual (Lebesgue) Banach space, equipped with the norm

$$
\|v\|_{0, p}:=\left(\int_{\Omega}|v(x)|^{p} d x\right)^{\frac{1}{p}} .
$$


The space $L_{\infty}(\Omega)$ with the norm

$$
\|v\|_{\infty}:=\operatorname{ess~sup}_{x \in \Omega}|v(x)|
$$

is the Banach space of all functions being essentially bounded in $\Omega$. Setting $p=2$, the space $L_{2}(\Omega)$ is a Hilbert space with the scalar product

$$
(u, v):=\int_{\Omega} u(x) \cdot v(x) d x
$$

and the norm

$$
\|v\|:=\|v\|_{0,2} \text {. }
$$

For $m \in \mathbb{N}_{0}$ and $1 \leq p<\infty$ let $H_{m, p}(\Omega)$ denote the Sobolev space of all functions $v \in L_{p}(\Omega)$ having distributional derivatives up to and including the order $m$ in $L_{p}(\Omega)$, and let

$$
\|v\|_{m, p}:=\left(\sum_{|\alpha|=0}^{m}\left\|\partial^{\alpha} v\right\|_{0, p}^{p}\right)^{\frac{1}{p}}
$$

denote the corresponding norm. The spaces $H_{m, p}(\Omega)$ are Banach spaces, for $p=2$ Hilbert spaces, and we define in this case $H_{m}(\Omega):=H_{m, 2}(\Omega)$ and

$$
\|v\|_{m}:=\|v\|_{m, 2}
$$

The closure of the space $C_{0}^{\infty}(\Omega)$ with respect to the norm $\|\cdot\|_{1}$ is defined by $\stackrel{\circ}{H}_{1}(\Omega)$, and the closure of the space $C_{0, \sigma}^{\infty}(\Omega)$ with respect to the norms $\|\cdot\|$ and $\|\cdot\|_{1}$ by $\mathcal{H}_{0}(\Omega)$ and $\mathcal{H}_{1}(\Omega)$, respectively.

The operator $P: L_{2}(\Omega) \rightarrow \mathcal{H}_{0}(\Omega)$ denotes the orthogonal projection such that

$$
L_{2}(\Omega)=\mathcal{H}_{0}(\Omega) \oplus\left\{v \in L_{2}(\Omega) \mid v=\nabla p \quad \text { for some } \quad p \in H_{1}(\Omega)\right\} .
$$

In $\stackrel{\circ}{H}_{1}(\Omega)$ and $\mathcal{H}_{1}(\Omega)$ we also use

$$
(\nabla u, \nabla v):=\sum_{i, j=1}^{3}\left(\partial_{j} u_{i}, \partial_{j} v_{i}\right), \quad\|\nabla v\|:=(\nabla v, \nabla v)^{\frac{1}{2}}
$$

as a scalar product and a norm $([13])$, respectively, as well as $(P \Delta u, P \Delta v)$ and $\|P \Delta v\|$ in $H_{2}(\Omega) \cap \mathcal{H}_{1}(\Omega)($ see $[4])$.

In the notation of the function spaces, the symbol $\Omega$ is sometimes omitted: $\mathcal{H}_{1}:=\mathcal{H}_{1}(\Omega), \ldots$ Throughout the paper, for the estimates we often use the continuity of the imbedding

$$
H_{j, p}(\Omega) \longrightarrow C^{m}(\bar{\Omega})
$$


valid for $j, m \in \mathbb{N}$ with $j>m+\frac{3}{p}$, and the compactness of the imbedding

$$
H_{m, r}(\Omega) \longrightarrow H_{j, p}(\Omega)
$$

valid for $1 \leq p, r<\infty$ and $j, m \in \mathbb{N}_{0}$ with $0 \leq j<m, \frac{3}{p}+m>\frac{3}{r}+j$ (see [1]).

Finally, by $C_{\Omega}, C_{M}, \ldots$ we denote positive constants depending on the terms indicated as subscripts. The values of theses constants may differ in different calculations.

Without loss of generality, throughout the paper we assume conservative external forces, i.e. in the system $\left(N_{0}\right)$ we set $F=0$.

\section{An initial value problem}

Let $J \subseteq \mathbb{R}$ denote a compact time interval and $v: J \times \bar{\Omega} \rightarrow \mathbb{R}^{3}$ a continuous velocity field. Moreover, for all $t \in J$ and $x \in \partial \Omega$ let $v(t, x)=0$, and assume $v(t):=v(t, \cdot) \in C^{l}(\bar{\Omega})$, $1 \leq l \leq 4$.

Consider for fixed $\left(s, x_{s}\right) \in J \times \bar{\Omega}$ in the time interval $J$ the initial value problem

$$
\begin{aligned}
\dot{x}(t) & =v(t, x(t)), \\
x(x) & =x_{s} .
\end{aligned}
$$

Here the function $t \rightarrow x(t)=X\left(t, s, x_{s}\right)$ denotes a solution of (2.4). It represents the trajectory of a particle of the fluid, which at initial time $t=s \in J$ is located in $x_{s} \in \bar{\Omega}$.

Due to $v(t, x)=0$ for $(t, x) \in J \times \partial \Omega$ we find that for all $\left(s, x_{s}\right) \in J \times \bar{\Omega}$ the solution $x(t)$ of (2.4) exists globally in $J$ and is uniquely determined there (see [16]).

For all $k=0,1, \ldots, l$ and all multi-indices $\alpha$ with $|\alpha|=0,1, \ldots, l$, respectively, the partial derivatives $\partial_{s}^{k} X, \partial_{t} \partial_{s}^{k} X, \partial^{\alpha} X$ and $\partial_{t} \partial^{\alpha} X$ exist and are continuous functions in $J \times J \times \bar{\Omega}$.

Due to the uniqueness of the solution, for the mappings

$$
X_{t, s}:\left\{\begin{aligned}
\bar{\Omega} & \rightarrow \bar{\Omega} \\
x & \rightarrow X_{t, s}(x):=X(t, s, x)
\end{aligned}\right.
$$

the composition rule

$$
X_{t, s} \circ X_{s, r}=X_{t, r}
$$

holds true for all $t, s, r \in J$. In particular, we find that for all $t, s \in J$ the mapping $X_{t, s}$ is a diffeomorphism in $\bar{\Omega}$, and we have

$$
X_{t, s}^{-1}=X_{s, t}
$$


Using $v(t, x)=0$ for $(t, x) \in J \times \partial \Omega$ we obtain, moreover, $X_{t, x}(\Omega)=\Omega$ for all $t, s \in J$.

Now consider Liouville's differential equation

$$
\partial_{t} \operatorname{det} \nabla X_{t, s}(x)=-\operatorname{div}_{X} v\left(t, X_{t, s}(X)\right) \operatorname{det} \nabla X_{t, s}(x)
$$

concerning the functional determinant $\operatorname{det} \nabla X_{t, s}(x)$.

If we assume $\operatorname{div} v(t, x)=0$ for all $(t, x) \in J \times \Omega$, then it follows

$$
\operatorname{det} \nabla X_{t, s}(x)=\operatorname{det} \nabla X_{s, s}(x)=\operatorname{det} \nabla x=1
$$

for all $t, s \in J$ and $x \in \Omega$.

Throughout this paper we call this important property of the mappings $X_{t, s}$ the conservation of measure.

It implies, in particular, that for divergence-free vector functions $v(t, \cdot)$ and all $t, s, r \in J$ we have

$$
\left\|v\left(t, X_{s, r}\right)\right\|_{o, p}=\|v(t)\|_{o, p}
$$

for all $1 \leq p \leq \infty$.

Lemma 2.1. Let $J \subseteq \mathbb{R}$ denote a compact time interval, and let $v \in C\left(J, H_{m}(\Omega) \cap \mathcal{H}_{1}(\Omega)\right)$ for $m \in\{3,4\}$ be given. Then we have

$$
a_{1}:=\max _{t \in J}\|v(t)\|_{\infty}<\infty, \quad a_{2}:=\max _{t \in J}\|\nabla v(t)\|_{\infty}<\infty,
$$

and for $m=4$, in addition,

$$
a_{3}:=\max _{t \in J}\left\|\nabla^{2} v(t)\right\|_{\infty}<\infty
$$

For every $\left(s, x_{s}\right) \in J \times \bar{\Omega}$ there is a uniquely determined solution $t \rightarrow x(t)=X\left(t, s, x_{s}\right)$ of the initial value problem (2.4), which exists in the whole $J$. For the mappings $X_{t, s}$ defined by (2.5) for all $t, s \in J$ we have the estimates

$$
\left\|X_{t, s}\right\|_{\infty} \leq|t-s| a_{1}+c_{\Omega}, \quad\left\|\nabla X_{t, s}\right\|_{\infty} \leq e^{|t-s| a_{2}}
$$

and for $m=4$, in addition,

$$
\left\|\nabla^{2} X_{t, s}\right\|_{\infty} \leq \frac{a_{3}}{a_{2}} e^{|t-s| a_{2}}\left(e^{|t-s| a_{2}}-1\right) .
$$

Here the constant $c_{\Omega}$ depends only on $\Omega$.

Proof: The existence of the norms $a_{i}$ follows from well-known imbedding theorems, as well as the existence and uniqueness of the solution of (2.4): For $l=m-2$ all the above 
required properties of $v$ are fulfilled, where the no-slip boundary condition and the vanishing divergence (solenoidality) follow from $v(t) \in \mathcal{H}_{1}(\Omega)$ for all $t \in J$.

Because for every half norm $\|\cdot\|_{h}$ and every absolutely continuous function $f: t \rightarrow f(t)$ the inequality $\frac{d}{d t}\|f(t)\|_{h} \leq\left\|f^{\prime}(t)\right\|_{h}$ holds true (see [15]), we obtain using the conservation of measure of the mappings $X_{t, s}$ for all $t, s \in J$ the following estimates:

$$
\begin{aligned}
\frac{d}{d t}\left\|X_{t, s}\right\|_{\infty} & \leq\left\|\partial_{t} X_{t, s}\right\|_{\infty}=\left\|v\left(t, X_{t, s}\right)\right\|_{\infty}=\|v(t)\|_{\infty}, \\
\frac{d}{d t}\left\|\nabla X_{t, s}\right\|_{\infty} & \leq\left\|\partial_{t} \nabla X_{t, s}\right\|_{\infty}=\left\|\nabla_{X} v\left(t, X_{t, s}\right) \nabla X_{t, s}\right\|_{\infty} \\
& \leq\|\nabla v(t)\|_{\infty} \cdot\left\|\nabla X_{t, s}\right\|_{\infty}, \\
\frac{d}{d t}\left\|\nabla^{2} X_{t, s}\right\|_{\infty} & \leq\left\|\partial_{t} \nabla^{2} X_{t, s}\right\|_{\infty}=\left\|\nabla_{X}^{2} v\left(t, X_{t, s}\right)\left(\nabla X_{t, s}\right)^{2}+\nabla_{X} v\left(t, X_{t, s}\right) \nabla^{2} X_{t, s}\right\|_{\infty} \\
& \leq\left\|\nabla^{2} v(t)\right\|_{\infty} \cdot\left\|\nabla X_{t, s}\right\|_{\infty}^{2}+\|\nabla v(t)\|_{\infty} \cdot\left\|\nabla^{2} X_{t, s}\right\|_{\infty} .
\end{aligned}
$$

These are three differential inequalities concerning the $L_{\infty}$-norms of the derivatives of the mappings $X_{t, s}$.

Due to $X_{s, s}\left(x_{s}\right)=x_{s}, \nabla X_{s, s}\left(x_{s}\right)=I$ and $\nabla^{2} X_{s, s}\left(x_{s}\right)=0$, where $I$ denotes the identity matrix and 0 the zero tensor for all $x_{s} \in \bar{\Omega}$, the corresponding initial values are also well known, and the estimates follow in both cases $s \leq t$ and $t \leq s$ from Gronwall's Lemma (see $[15])$.

\section{Approximation of the convective term}

Up to now it is not known wether the Navier-Stokes initial boundary value problem $\left(N_{0}\right)$ in three dimensions is well-posed or not: We only know the existence and uniqueness of a strong solution locally in time. So in the following we want to derive a suitable smoothing procedure to end up with a modified Navier-Stokes-like system of equations, which can even be solved globally in time.

This system of equations $\left(\tilde{N}_{\varepsilon}\right)$ depends on a certain regularizing parameter $\varepsilon>0$ in the nonlinear term. In the following we shall develop this regularized system step by step.

To do so, let us first recall the physical deduction of the Navier-Stokes equations: The nonlinear convective term $v(t, x) \cdot \nabla v(t, x)$, which is responsable for the non-global solvability of these equations, results from the total derivative of the velocity field $v(t, x)$. Thus a socalled total or Lagrangian difference quotient could be used for an approximation of the nonlinear convective term: 
Definition 3.1. Let $J \subseteq \mathbb{R}$ denote a compact time interval and let $v \in C\left(J, H_{3}(\Omega) \cap \mathcal{H}_{1}(\Omega)\right)$ be given. Let $\varepsilon>0$ and let $t, s, s+\varepsilon \in J, x \in \bar{\Omega}$. Then the quotients

$$
\frac{1}{\varepsilon}\left\{v\left(t, X_{s+\varepsilon, s}(x)\right)-v(t, x)\right\}
$$

and

$$
\frac{1}{\varepsilon}\left\{v(t, x)-v\left(t, X_{s, s+\varepsilon}(x)\right)\right\}
$$

are well defined and denoted by an upwards and a backwards total (Lagrangian) difference quotient, respectively. Summing up both quotients and dividing by two we obtain

$$
\frac{1}{2 \varepsilon}\left\{v\left(t, X_{s+\varepsilon, s}(x)\right)-v\left(t, X_{s, s+\varepsilon}(x)\right)\right\}
$$

and call it a central total (Lagrangian) difference quotient.

Remark 3.2. Using a mean value theorem, as $\varepsilon \rightarrow 0$ all the above mentioned difference quotients converge to $v(s, x) \cdot \nabla v(t, x)$. For example, for the upwards quotient we find

$$
\begin{aligned}
\frac{1}{\varepsilon}\left\{v\left(t, X_{s+\varepsilon, s}(x)\right)-v(t, x)\right\} & =\frac{1}{\varepsilon}\left\{v\left(t, X_{s+\varepsilon, s}(x)\right)-v\left(t, X_{s, s}(x)\right)\right\} \\
& =\frac{1}{\varepsilon} \int_{s}^{s+\varepsilon} \partial_{\tau} X_{\tau, s}(x) \cdot \nabla v\left(t, X_{\tau, s}(x)\right) d \tau \\
& =\frac{1}{\varepsilon} \cdot \int_{s}^{s+\varepsilon} v\left(\tau, X_{\tau, s}(x)\right) \cdot \nabla v\left(t, X_{\tau, s}(x)\right) d \tau
\end{aligned}
$$

where the term on the right hand side tends to $v\left(s, X_{s, s}(x)\right) \cdot \nabla v\left(t, X_{s, s}(x)\right)=v(s, x) \cdot \nabla v(t, x)$ as $\varepsilon \rightarrow 0$.

It is well known (see [13]) that for vector functions $u \in \mathcal{H}_{1}(\Omega), w \in \stackrel{\circ}{H}_{1}(\Omega)$ the orthogonality relation $(u \cdot \nabla w, w)=0$ holds true. This important relation is used by Hopf (see [5]) to prove the existence of weak solutions of the Navier-Stokes equations $\left(N_{0}\right)$ global in time. Using an approximation of the convective term $v(t, x) \cdot \nabla v(t, x)$ by a central total difference quotient, we can prove the following analogue of this orthogonality relation:

Lemma 3.3. Under the assumptions of Definition 3.1, for the central total difference quotient the following orthogonality relation holds true:

$$
\left(\frac{1}{2 \varepsilon}\left[v\left(t, X_{s+\varepsilon, s}(\cdot)\right)-v\left(t, X_{s, s+\varepsilon}(\cdot)\right)\right], v(t, \cdot)\right)=0 .
$$

Proof: For all $s_{1}, s_{2} \in J$ the mappings $X_{s_{1}, s_{2}}$ are measure conserving, and by (2.6), we obtain $X_{s_{1}, s_{2}} \circ X_{s_{2}, s_{1}}(x)=x$ for all $x \in \bar{\Omega}$. Using the symmetry of the scalar product this 
implies the orthogonality:

$$
\begin{aligned}
& \left(v\left(t, X_{s+\varepsilon, s}(\cdot)\right)-v\left(t, X_{s, s+\varepsilon}(\cdot)\right), v(t, \cdot)\right) \\
= & \left(v\left(t, X_{s+\varepsilon, s}(\cdot)\right), v(t, \cdot)\right)-\left(v\left(t, X_{s, s+\varepsilon} \circ X_{s+\varepsilon, s}(\cdot)\right), v\left(t, X_{s+\varepsilon, s}(\cdot)\right)\right) \\
= & 0 .
\end{aligned}
$$

Remark 3.4. From Lemma 3.3 it follows that for sufficiently regular solutions of an approximate system regularized by central total differences the energy equation is satisfied. As seen from the proof above, this important equation does not hold true if only a one-sided total difference quotient is used to approximate the convective term.

To avoid fixed point considerations - it is clear that in general both the velocity field $v$ as well as the trajectories $X$ are not known - in the following we use in addition a time delay: The convective term $v(t, x) \cdot \nabla v(t, x)$ is replaced by a central total difference quotient of the form

$$
\frac{1}{2 \varepsilon}\left\{v\left(t, X_{s+\varepsilon, s}(x)\right)-v\left(t, X_{s, s+\varepsilon}(x)\right)\right\}
$$

with times $s, s+\varepsilon<t$. In these time points - using a step by step construction - the velocity field is known, already.

Let us now formulate the regularized problem $\left(\tilde{N}_{\varepsilon}\right)$ as follows:

Let $T \in \mathbb{R}(T>0)$ and $N \in \mathbb{N}(N \geq 2)$ be given. Setting $\varepsilon:=\frac{T}{N}>0$ we define by $t_{k}=k \varepsilon$ $(k=0, \pm 1, \ldots, \pm N)$ an equidistant time grid on the compact time interval $[-T,+T]$.

Construct a velocity field $v=\left(v_{1}, v_{2}, v_{3}\right)$ and some pressure function $p$ as solution of the regularized Navier-Stokes initial boundary value problem

$$
\partial_{t} v-\nu \Delta v+\nabla p=-Z_{\varepsilon} v
$$

$$
\begin{array}{rlr}
\nabla \cdot v & =0 & (t, x) \in(0, T] \times \Omega \\
v_{\mid \partial \Omega} & =0 \\
\partial_{t} v_{\left.\right|_{t=0}} & =f . &
\end{array}
$$

Here for $(t, x) \in\left[t_{k}, t_{k+1}\right] \times \bar{\Omega}$ and $k=0,1, \ldots, N-1$ we define

$$
\begin{aligned}
Z_{\varepsilon} v(t, x):= & Z_{\varepsilon}^{k} v(t, x) \\
:= & \frac{t-t_{k}}{\varepsilon} \cdot \frac{1}{2 \varepsilon} \cdot\left\{v\left(t, X_{t_{k}, t_{k-1}}(x)\right)-v\left(t, X_{t_{k-1}, t_{k}}(x)\right)\right\}+ \\
& \frac{t_{k+1}-t}{\varepsilon} \cdot \frac{1}{2 \varepsilon} \cdot\left\{v\left(t, X_{t_{k-1}, t_{k-2}}(x)\right)-v\left(t, X_{t_{k-2}, t_{k-1}}(x)\right)\right\},
\end{aligned}
$$

where the mappings $X_{t_{i}, t_{j}}$ have to be constructed from the solution $t \rightarrow x(t)=X\left(t, s, x_{s}\right)$ of a corresponding initial value problem (2.4) with a velocity field already known. 
Remark 3.5. To compute the solution $v(t)$ of $\left(\tilde{N}_{\varepsilon}\right)$ in the first subinterval $\left[t_{0}, t_{1}\right]$ we have to construct the mappings $X_{t_{0}, t_{-1}}$ and $X_{t_{-1}, t_{-2}}$ together with the inverse mappings $X_{t_{0}, t_{-1}}^{-1}=$ $X_{t_{-1}, t_{0}}$ and $X_{t_{-1}, t_{-2}}^{-1}=X_{t_{-2}, t_{-1}}$, respectively. This construction will be carried out in the next section.

Remark 3.6. The global construction step by step requires certain regularity properties of the solution $v(t)$ of $\left(\tilde{N}_{\varepsilon}\right)$ on the subintervals $J_{k}:=\left[t_{k}, t_{k+1}\right]$ for $\left.k=0,1, \ldots, N-1\right)$. These regularity properties are necessary to imply the unique solvability of the initial value problem (2.4) in $J_{k}$ and thus the existence of the mappings $X_{t_{k+1}, t_{k}}, X_{t_{k}, t_{k-1}}$, which are needed for the construction of the solution on subsequent time intervals.

This high degree of regularity - $v \in C\left(J_{k}, H_{3}(\Omega) \cap \mathcal{H}_{1}(\Omega)\right)$ is sufficient, but $v \in C\left(J_{k}, H_{2}(\Omega)\right.$ $\cap \mathcal{H}_{1}(\Omega)$ ) is not sufficient - leads to compatibility conditions arising on the boundary of the time-space cylinder (see [4] and [14]), as usual for parabolic problems. In our case, due to the stepwise construction, these conditions appear in the points $\left(t_{k}, x\right)$ with $k=0,1, \ldots, N-1$ and $x \in \partial \Omega$.

The compatibility conditions for $t_{k}>0$ are fulfilled because of the continuity of the functions $Z_{\varepsilon} v(\cdot, x)$ in $[0, T]$ for $x \in \bar{\Omega}$, and the condition at time $t_{0}=0$ holds by a special initial construction using an idea of Solonnikov: Instead of the initial velocity $v(0)$ the initial acceleration $\partial_{t} v(0)$ has to be prescribed in a suitable way. This construction is carried out in the next section.

\section{Construction of the initial data}

Let $T>0,2 \leq N \in \mathbb{N}, \varepsilon=\frac{T}{N}>0$ and $t_{k}=k \varepsilon$ for $k=0, \pm 1, \ldots, \pm N$ as fixed above in problem $\left(\tilde{N}_{\varepsilon}\right)$.

Let $\bar{v} \in C\left([-T, 0], H_{m}(\Omega) \cap \mathcal{H}_{1}(\Omega)\right)$ for $m \in\{3,4\}$ be given. Then by Lemma 2.1 it follows that the mapping

$$
X_{t_{-1}, t_{-2}}: \begin{cases}\bar{\Omega} & \rightarrow \bar{\Omega} \\ x & \rightarrow X_{t_{-1}, t_{-2}}(x)=X(-\varepsilon,-2 \varepsilon, x)\end{cases}
$$

defined by (2.5) exists, as well as its inverse

$$
X_{t_{-2}, t_{-1}}: \begin{cases}\bar{\Omega} & \rightarrow \bar{\Omega} \\ x & \rightarrow X_{t_{-2}, t_{-1}}(x)=X(-2 \varepsilon,-\varepsilon, x) .\end{cases}
$$

Denoting by $P: L_{2}(\Omega) \rightarrow \mathcal{H}_{0}(\Omega)$ the orthogonal projection (see [13]) we consider in $\Omega$ the stationary regularized Navier-Stokes boundary value problem

$$
\nu P \Delta v_{0}-\frac{1}{2 \varepsilon} P\left\{v_{0} \circ X_{t_{-1}, T_{-2}}-v_{0} \circ X_{t_{-2}, t_{-}}\right\}=P f .
$$


Lemma 4.1. Let $\bar{v} \in C\left([-T, 0], H_{m}(\Omega) \cap \mathcal{H}_{1}(\Omega)\right)$ for $m \in\{3,4\}$, and let the mappings $X_{t_{-1}, t_{-2}}, X_{t_{-2}, t_{-1}}$ be constructed as above. Let $f \in H_{m-2}(\Omega) \cap \mathcal{H}_{1}(\Omega)$. Then there exists a uniquely determined solution $v_{0} \in H_{m}(\Omega) \cap \mathcal{H}_{1}(\Omega)$ of problem (4.3).

Proof: Let us set $X:=X_{t_{-1}, t_{-2}}$ for abbreviation. Then from the linearity of the problem, for the difference $w_{0}:=v_{0}^{1}-v_{0}^{2}$ of two solutions $v_{0}^{1}$ and $v_{0}^{2}$ it follows the identity

$$
\nu P \Delta w_{0}-\frac{1}{2 \varepsilon} P\left\{w_{0} \circ X-w_{0} \circ X^{-1}\right\}=0 .
$$

Here we find $\left(w_{0} \circ X-w_{0} \circ X^{-1}, w_{0}\right)=0$ due to the measure conserving property of the mappings $X$, and the uniqueness follows from the inequality of Poincaré:

$$
\nu\left\|w_{0}\right\|^{2} \leq \nu c_{\Omega}\left\|\nabla w_{0}\right\|^{2}=0 .
$$

The existence of a solution $v_{0} \in \mathcal{H}_{1}(\Omega)$ can be obtained from the theory of the stationary Navier-Stokes equations (see [13]), and for the regularity statement $v_{0} \in H_{m}(\Omega)$ we can use the estimate of Cattabriga ([2]), which means that we only have to show $\left\|v_{0} \circ X\right\|_{m-2}<\infty$, $\left\|v_{0} \circ X^{-1}\right\|_{m-2}<\infty$. This indeed follows from $v_{0} \in \mathcal{H}_{1}(\Omega)$ and the regularity properties of the mappings $X$ and $X^{-1}$ following Lemma 2.1.

Now using the function $\bar{v}$ prescribed above together with the solution $v_{0}$ of the system (4.3) we can define for example by linear interpolation some function $\tilde{v} \in C\left([-T, 0], H_{m}(\Omega) \cap \mathcal{H}_{1}(\Omega)\right)$ for $m \in\{3,4\}$ :

$$
\tilde{v}(t):=\left\{\begin{array}{crr}
\bar{v}(t) & & t \in[-T,-\varepsilon] \\
& \text { for } & \\
\frac{1}{\varepsilon}\left\{(t+\varepsilon) v_{0}-t \cdot \bar{v}(-\varepsilon)\right\} & & t \in[-\varepsilon, 0] .
\end{array}\right.
$$

Now we use the function $\tilde{v}$ and Lemma 2.1 to construct the mapping

$$
X_{t_{0}, t_{-1}}: \begin{cases}\bar{\Omega} & \rightarrow \bar{\Omega} \\ x & \rightarrow X_{t_{0}, t_{-1}}(x)=X(0,-\varepsilon, x)\end{cases}
$$

together with its inverse

$$
X_{t_{-1}, t_{0}}:\left\{\begin{array}{l}
\bar{\Omega} \rightarrow \bar{\Omega} \\
x \rightarrow X_{t_{-1}, t_{0}}(x)=X(-\varepsilon, 0, x) .
\end{array}\right.
$$

All the mappings (4.1), (4.2), (4.5) and (4.6) constructed in this way are used in the next section where we start the investigation of problem $\left(\tilde{N}_{\varepsilon}\right)$ on the first subinterval $\left[t_{0}, t_{1}\right]$. Due to Lemma 4.1 we can replace the initial condition $\partial_{t} v_{\left.\right|_{t=0}}=f$ from problem $\left(\tilde{N}_{\varepsilon}\right)$ (note we assume $f \in H_{m-2}(\Omega) \cap \mathcal{H}_{1}(\Omega)$ with $\left.m \in\{3,4\}\right)$ by the initial condition $v_{\mid t=0}=v_{0}$ (in this case we have $\left.v_{0} \in H_{m}(\Omega) \cap \mathcal{H}_{1}(\Omega)\right)$.

Moreover, the above construction implies that the initial acceleration $\partial_{t} v(0)=f$ is contained in $\mathcal{H}_{1}(\Omega)$, if the differential equations still hold for $t=0$. This ensures - as we shall see later on - that the important compatibility condition at time $t=0$ is satisfied. 


\section{Strongly $H_{2}$-continuous solutions}

We consider the problem $\left(\tilde{N}_{\varepsilon}\right)$ restricted to $t \in\left[t_{0}, t_{1}\right]=[0, \varepsilon]$ in the following form:

Find a velocity field $v=\left(v_{1}, v_{2}, v_{3}\right)$ and some pressure function $p$ as a solution of the regularized equations

$$
\begin{aligned}
\partial_{t} v-\nu \Delta v+\nabla p & =-Z_{\varepsilon}^{0} v \\
\nabla \cdot v & =0 \\
v_{\left.\right|_{\partial \Omega}} & =0 \\
v_{\left.\right|_{t=0}} & =v_{0} .
\end{aligned}
$$

$$
(t, x) \in(0, \varepsilon] \times \Omega
$$

Here for $(t, x) \in[0, \varepsilon] \times \bar{\Omega}$ we define

$$
Z_{\varepsilon}^{0} v(t, x):=\frac{t}{2 \varepsilon^{2}}\left\{v(t, X(x))-v\left(t, X^{-1}(x)\right)\right\}+\frac{\varepsilon-t}{2 \varepsilon^{2}}\left\{v(t, Y(x))-v\left(t, Y^{-1}(x)\right)\right\}
$$

with some given measure conserving homomorphisms $X: \bar{\Omega} \rightarrow \bar{\Omega}$ and $Y: \bar{\Omega} \rightarrow \bar{\Omega}$. The function $v_{0} \in H_{2}(\Omega) \cap \mathcal{H}_{1}(\Omega)$ is a given initial velocity distribution.

In this section we show the existence of a solution $t \rightarrow v(t):=v(t, \cdot)$ to the linear system $\left(N_{\varepsilon}^{0}\right)$ being strongly $H_{2}$-continuous in $[0, \varepsilon]$. This solution is uniquely determined and satisfies the energy equation (Theorem 5.7). Similar to [3] we prove the existence with help of a Galerkin ansatz based on the eigenfunctions of the Stokes operator $-P \Delta$. This is done in the following way: First we derive suitable a-priori estimates for the Galerkin approximations and then we use compactness arguments to proceed to the limit and prove the existence of a solution.

\section{(a) Galerkin ansatz}

The Stokes operator $-P \Delta: H_{2}(\Omega) \cap \mathcal{H}_{1}(\Omega) \rightarrow \mathcal{H}_{0}(\Omega)$ defines in $\mathcal{H}_{1}(\Omega)$ a symmetric positive definite operator with compact inverse $(-P \Delta)^{-1}: \mathcal{H}_{0}(\Omega) \rightarrow \mathcal{H}_{0}(\Omega)$ (see [12]). Hence there is a sequence $\left(\lambda_{i}\right)_{i}$ of positive eigenvalues satisfying $0<\lambda_{1} \leq \lambda_{2} \leq \ldots \leq \lambda_{n} \rightarrow \infty$, and the corresponding sequence $\left(e_{i}\right)_{i}$ of eigenfunctions represents a complete orthonormal system in $\mathcal{H}_{0}(\Omega)$. Due to the regularity of the boundary $\left(\partial \Omega \in C^{4}\right)$ we have $e_{i} \in H_{4}(\Omega)$ for all $i \in \mathbb{N}$ (see [13]).

Now for $i=1,2, \ldots, n(n \in \mathbb{N})$ and $t>0$ we consider the following initial value problem for ordinary differential equations:

$$
\begin{aligned}
& c_{\mathrm{in}}^{\prime}(t)=-\nu \lambda_{i} c_{\mathrm{in}}(t)-\frac{1}{2 \varepsilon^{2}} \sum_{j=1}^{n} c_{\mathrm{jn}}(t) \cdot\left(t\left\{e_{j} \circ X-e_{j} \circ X^{-1}\right\}+(\varepsilon-t)\left\{e_{j} \circ Y-e_{j} \circ Y^{-1}\right\}, e_{i}\right), \\
& c_{\mathrm{in}}(0)=\left(v_{0}, e_{i}\right) .
\end{aligned}
$$

This is a linear system for the functions $c_{\text {in }}$, and it is globally and uniquely solvable with $c_{\text {in }} \in C^{\infty}([0, \varepsilon])$ for all $i=1, \ldots, n$. 
The function

$$
v^{n}:\left\{\begin{array}{l}
{[0, \varepsilon] \times \bar{\Omega} \rightarrow \mathbb{R}^{3}} \\
(t, x) \rightarrow v^{n}(t, x):=\sum_{i=1}^{n} c_{\text {in }}(t) \cdot e_{i}(x)
\end{array}\right.
$$

is denoted as Galerkin approximation of order $n$ for the solution $v$ of the problem $\left(N_{\varepsilon}^{0}\right)$.

Due to the construction, for the functions $v^{n}(t):=v^{n}(t, \cdot)$ for all $t \in(0, \varepsilon]$ and all $i=$ $1,2, \ldots, n$ the following equations hold true:

$$
\begin{gathered}
\left(\partial_{t} v^{n}(t), e_{i}\right)-\nu\left(P \Delta v^{n}(t), e_{i}\right)=-\left(Z_{\varepsilon}^{0} v^{n}(t), e_{i}\right) \\
v^{n}(0)=\sum_{j=1}^{n}\left(v_{0}, e_{j}\right) e_{j} .
\end{gathered}
$$

\section{(b) Estimates of the Galerkin approximations}

All estimates of the Galerkin approximations $v^{n}(t)$ can be obtained from the following two lemmata and are valid - depending on the regularity of the data - uniformly for $t \in[0, \varepsilon]$ or only for $t \in(0, \varepsilon]$.

Lemma 5.1. Let $k, n \in \mathbb{N}$. Then for the Galerkin approximation $v^{n}(t)$ defined for $t \in[0, \varepsilon]$ by (5.2) the following identities hold true:

$$
\begin{aligned}
\frac{d}{d t}\left\|v^{n}(t)\right\|^{2}+2 \nu\left\|\nabla v^{n}(t)\right\|^{2}= & 0 \\
\frac{d}{d t}\left\|\partial_{t}^{k} v^{n}(t)\right\|^{2}+2 \nu\left\|\nabla \partial_{t}^{k} v^{n}(t)\right\|^{2}= & -2\left(\partial_{t}^{k} Z_{\varepsilon}^{0} v^{n}(t), \partial_{t}^{k} v^{n}(t)\right) \\
= & -\frac{k}{\varepsilon^{2}}\left(\partial_{t}^{k-1} v^{n}(t, X)-\partial_{t}^{k-1} v^{n}\left(t, X^{-1}\right)\right. \\
& \left.-\partial_{t}^{k-1} v^{n}(t, Y)+\partial_{t}^{k-1} v^{n}\left(t, Y^{-1}\right), \partial_{t}^{k} v^{n}(t)\right) \\
\frac{d}{d t}\left\|\nabla v^{n}(t)\right\|^{2}+2 \nu\left\|P \Delta v^{n}(t)\right\|^{2}= & -2\left(Z_{\varepsilon}^{0} v^{n}(t),-P \Delta v^{n}(t)\right) \\
\frac{d}{d t}\left\|\nabla \partial_{t}^{k} v^{n}(t)\right\|^{2}+2 \nu\left\|P \Delta \partial_{t}^{k} v^{n}(t)\right\|^{2}= & -2\left(\partial_{t}^{k} Z_{\varepsilon}^{0} v^{n}(t),-P \Delta \partial_{t}^{k} v^{n}(t)\right) \\
= & -2\left(Z_{\varepsilon}^{0} \partial_{t}^{k} v^{n}(t),-P \Delta \partial_{t}^{k} v^{n}(t)\right) \\
& -\frac{k}{\varepsilon^{2}}\left(\partial_{t}^{k-1} v^{n}(t, X)-\partial_{t}^{k-1} v^{n}\left(t, X^{-1}\right)\right. \\
& \left.-\partial_{t}^{k-1} v^{n}(t, Y)+\partial_{t}^{k-1} v^{n}\left(t, Y^{-1}\right),-P \Delta \partial_{t}^{k} v^{n}(t)\right) .
\end{aligned}
$$


Proof: Let $k \geq 0$. Then the first sequence of identities in the second line of (5.5) and (5.6), respectively, follows from differentiating the equation (5.3) $k$ times with respect to $t$, multiplying the result scalar by $\partial_{t}^{k} c_{\text {in }}(t)$ and $\lambda_{i} \partial_{t}^{k} c_{\text {in }}(t)$, respectively, and afterwards summing up for $i=1, \ldots, n$. Concerning the first line of (5.5) we use in addition the orthogonality $\left(Z_{\varepsilon}^{0} v^{n}(t), v^{n}(t)\right)=0$, which follows from the measure conserving property of the mappings $X, Y$.

The second sequence of identities is obtained from

$$
\begin{aligned}
\partial_{t}^{k} Z_{\varepsilon}^{0} v^{n}(t)= & \partial_{t}^{k-1}\left(Z_{\varepsilon}^{0} \partial_{t} v^{n}(t)+\frac{1}{2 \varepsilon^{2}}\left\{v^{n}(t, X)-v^{n}\left(t, X^{-1}\right)-v^{n}(t, Y)+v^{n}\left(t, Y^{-1}\right)\right\}\right) \\
= & \cdots \\
= & Z_{\varepsilon}^{0} \partial_{t}^{k} v^{n}(t)+\frac{k}{2 \varepsilon^{2}} \cdot \\
& \left\{\partial_{t}^{k-1} v^{n}(t, X)-\partial_{t}^{k-1} v^{n}\left(t, X^{-1}\right)-\partial_{t}^{k-1} v^{n}(t, Y)+\partial_{t}^{k-1} v^{n}\left(t, Y^{-1}\right)\right\} .
\end{aligned}
$$

Estimating the right hand sides in (5.5) and (5.6) using the inequalities of Hölder, Poincaré und Young (see [7]), the measure conserving property of the mappings $X$ and $Y$, and, finally, the absorbtion of terms arising on both sides of the inequalities, the following corollary can be proved:

Corollary 5.2. Let $k, n \in \mathbb{N}$. Then the Galerkin approximations $v^{n}(t)$ defined for $t \in[0, \varepsilon]$ by (5.2) satisfy the following differential inequalities:

$$
\begin{aligned}
\frac{d}{d t}\left\|\partial_{t}^{k} v^{n}(t)\right\|^{2}+\nu\left\|\nabla \partial_{t}^{k} v^{n}(t)\right\|^{2} & \leq c_{\Omega, \nu, \varepsilon, k}\left\|\partial_{t}^{k-1} v^{n}(t)\right\|^{2}, \\
\frac{d}{d t}\left\|\nabla v^{n}(t)\right\|^{2}+\nu\left\|P \Delta v^{n}(t)\right\|^{2} & \leq c_{\nu, \varepsilon}\left\|v^{n}(t)\right\|^{2}, \\
\frac{d}{d t}\left\|\nabla \partial_{t}^{k} v^{n}(t)\right\|^{2}+\nu\left\|P \Delta \partial_{t}^{k} v^{n}(t)\right\|^{2} & \leq c_{\nu, \varepsilon, k}\left(\left\|\partial_{t}^{k-1} v^{n}(t)\right\|^{2}+\left\|\partial_{t}^{k} v^{n}(t)\right\|^{2}\right) .
\end{aligned}
$$

Finally, from the Galerkin equations (5.3) we quote immediately:

Lemma 5.3. Let $k, n \in \mathbb{N}$. Then for the Galerkin approximation $v^{n}(t)$ defined by (5.2) for $t \in[0, \varepsilon]$ the following inequalities hold true:

$$
\begin{aligned}
\left\|\partial_{t} v^{n}(t)\right\|^{2} & \leq c_{\nu, \varepsilon}\left(\left\|P \Delta v^{n}(t)\right\|^{2}+\left\|v^{n}(t)\right\|^{2}\right) \\
\left\|\partial_{t}^{k+1} v^{n}(t)\right\|^{2} & \leq c_{\nu, \varepsilon, k}\left(\left\|P \Delta \partial_{t}^{k} v^{n}(t)\right\|^{2}+\left\|\partial_{t}^{k} v^{n}(t)\right\|+\left\|\partial_{t}^{k-1} v^{n}(t)\right\|^{2}\right), \\
\left\|P \Delta v^{n}(t)\right\|^{2} & \leq c_{\nu, \varepsilon}\left(\left\|\partial_{t} v^{n}(t)\right\|^{2}+\left\|v^{n}(t)\right\|^{2}\right), \\
\left\|P \Delta \partial_{t}^{k} v^{n}(t)\right\|^{2} & \leq c_{\nu, \varepsilon, k}\left(\left\|\partial_{t}^{k+1} v^{n}(t)\right\|^{2}+\left\|\partial_{t}^{k} v^{n}(t)\right\|^{2}+\left\|\partial_{t}^{k-1} v^{n}(t)\right\|^{2}\right) .
\end{aligned}
$$


Now we are ready to prove the above mentioned a-priori estimates for the Galerkin approximations $v^{n}(t)$ for $t \in[0, \varepsilon]$ :

Lemma 5.4. Let $n \in \mathbb{N}$. Then the Galerkin approximation $v^{n}(t)$ defined by (5.2) satisfies for all $t \in[0, \varepsilon]$ the following a-priori estimates:

$$
\begin{gathered}
\left\|v^{n}(t)\right\|^{2}+2 \nu \int_{0}^{t}\left\|\nabla v^{n}(\tau)\right\|^{2} d \tau=\left\|v^{n}(0)\right\|^{2} \leq\left\|v_{0}\right\|^{2} \\
\qquad \nabla v^{n}(t)\left\|^{2}+\nu \int_{0}^{t}\right\| P \Delta v^{n}(\tau) \|^{2} d \tau \\
\leq\left\|\nabla v^{n}(0)\right\|^{2}+c_{\nu, \varepsilon}\left\|v_{0}\right\|^{2} t \\
\qquad \partial_{t} v^{n}(t)\left\|^{2}+\nu v_{0}\right\|^{2}+c_{\nu, \varepsilon}\left\|v_{0}\right\|^{2} t \\
\int_{0}^{t}\left\|\nabla \partial_{\tau} v^{n}(\tau)\right\|^{2} d \tau \leq\left\|\partial_{t} v^{n}(0)\right\|^{2}+c_{\Omega, \nu, \varepsilon}\left\|v_{0}\right\|^{2} t \\
\left\|\partial_{t} v^{n}(0)\right\|^{2} \leq c_{\Omega, \nu, \varepsilon}\left\|P \Delta v_{0}\right\|^{2} \\
\left\|P \Delta v^{n}(t)\right\|^{2} \leq c_{\Omega, \nu, \varepsilon}\left\|P \Delta v_{0}\right\|^{2}
\end{gathered}
$$

Here all appearing constants are independent of $n$.

Proof: The estimates (5.12), (5.13), (5.14) follow by integration from (5.5), (5.9) and (5.8). The estimates (5.15) and (5.16) can be obtained from (5.10) and (5.11), using the estimate of Cattabriga in the form

$$
\|w\|^{2} \leq c_{\Omega}\|P \Delta w\|^{2}
$$

valid for functions $w \in H_{2}(\Omega) \cap \mathcal{H}_{1}(\Omega)$ (compare [2] and [4]).

In Lemma 5.4 all norm estimates of the Galerkin approximations $v^{n}(t)$, which are valid uniformly for all $t \in[0, \varepsilon]$, are listed. Due to the regularity of the initial value $v_{0} \in H_{2}(\Omega) \cap$ $\mathcal{H}_{1}(\Omega)$, higher order estimates uniformly in time cannot be expected.

Nevertheless, higher order regularity statements about the solution of $\left(N_{\varepsilon}^{0}\right)$ can be proved, if norm estimates for higher order derivatives of the functions $v^{n}(t)$ independent of $n$ are available. This is only possible for $t>0$ or uniformly for $t \in[\alpha, \varepsilon]$ with $\alpha>0$ : 
Lemma 5.5. Let $\alpha \in \mathbb{R}$ with $0<\alpha<\varepsilon$, and let $n \in \mathbb{N}, k \in \mathbb{N}_{0}$. Then for the Galerkin approximation $v^{n}(t)$ defined by (5.2) the following estimates hold true for all $t \in[\alpha, \varepsilon]$ :

$$
\begin{aligned}
\left\|\partial_{t}^{k} v^{n}(t)\right\|^{2}+2 \nu \int_{\alpha}^{t}\left\|\nabla \partial_{\tau}^{k} v^{n}(\tau)\right\|^{2} d \tau & \leq K_{1}, \\
\left\|\nabla \partial_{t}^{k} v^{n}(t)\right\|^{2}+\nu \int_{\alpha}^{t}\left\|P \Delta \partial_{\tau}^{k} v^{n}(\tau)\right\|^{2} d \tau & \leq K_{2}, \\
\left\|P \Delta \partial_{t}^{k} v^{n}(t)\right\|^{2} & \leq K_{3} .
\end{aligned}
$$

Here the constants $K_{1}, K_{2}, K_{3}$ depend only on $\alpha, \varepsilon, \nu, k, \Omega$ and on the $H_{2}$-Norm of the initial value $v_{0}$, but not on $n$.

The proof follows as in [3] by combining mean value theorems with methods of bootstrapping to increase the regularity.

At the end of this subsection we shall prove a continuity statement which is needed for later use. Taking into account (5.12) and (5.13), we find that for the function $v^{n}(t)$ the inequalities

$$
\limsup _{t \searrow 0}\left\|v^{n}(t)\right\| \leq\left\|v^{n}(0)\right\|
$$

and

$$
\limsup _{t \searrow 0}\left\|\nabla v^{n}(t)\right\| \leq\left\|\nabla v^{n}(0)\right\| .
$$

hold true, respectively. A corresponding statement for the norm $\left\|P \Delta v^{n}(t)\right\|$ can be obtained from the next lemma:

Lemma 5.6. Let $n \in \mathbb{N}$. Then for the Galerkin approximation $v^{n}(t)$ defined by (5.2) for all $t \in[0, \varepsilon]$ the following inequality holds:

$$
\begin{gathered}
\left\|P \Delta v^{n}(t)\right\|^{2}+\frac{2}{\nu} \int_{0}^{t}\left\|\nabla \partial_{\tau} v^{n}(\tau)\right\|^{2} d \tau \\
\leq\left\|P \Delta v^{n}(0)\right\|^{2}+K t+c_{\varepsilon, \nu}\left(v^{n}(\tau, Y(\cdot))-v^{n}\left(\tau, Y^{-1}(\cdot)\right), P \Delta v^{n}(\tau, \cdot)\right) \mid \begin{array}{l}
\tau=t \\
\tau=0
\end{array} .
\end{gathered}
$$

Here the constant $K$ depends only on $\Omega, \varepsilon, \nu$ and the $H_{2}$-norm of the initial value $v_{0}$. 
Proof: From (5.3) we obtain by multiplication with $\lambda_{i} c_{\text {in }}^{\prime}(t)$, by summing up for $i=1, \ldots, n$ and by integration from 0 to $t$ the identity

$$
\int_{0}^{t}\left\|\nabla \partial_{\tau} v^{n}(\tau)\right\|^{2} d \tau+\frac{\nu}{2}\left(\left\|P \Delta v^{n}(t)\right\|^{2}-\left\|P \Delta v^{n}(0)\right\|^{2}\right)=-\int_{0}^{t}\left(Z_{\varepsilon}^{0} v^{n}(\tau),-P \Delta \partial_{\tau} v^{n}(\tau)\right) d \tau .
$$

Using partial integration and (5.7) it follows for the right hand side

$$
\begin{aligned}
& \left(Z_{\varepsilon}^{0} v^{n}(\tau), P \Delta v^{n}(\tau)\right) \mid \begin{array}{l}
\tau=t \\
\tau=0
\end{array}+\int_{0}^{t}\left(Z_{\varepsilon}^{0} \partial_{\tau} v^{n}(\tau),-P \Delta v^{n}(\tau)\right) d \tau \\
& +\frac{1}{2 \varepsilon^{2}} \int_{0}^{t}\left(v^{n}(\tau, X)-v^{n}\left(\tau, X^{-1}\right)-v^{n}(\tau, Y)\right. \\
& \left.+v^{n}\left(\tau, Y^{-1}\right),-P \Delta v^{n}(\tau)\right) d \tau=: \sum_{i=1}^{3} s_{i} .
\end{aligned}
$$

Finally, the terms $s_{1}, s_{2}, s_{3}$ can be estimated using (5.14) and (5.16):

$$
\begin{aligned}
& s_{1} \leq K t+c_{\varepsilon}\left(v^{n}(\tau, Y)-v^{n}\left(\tau, Y^{-1}\right), P \Delta v^{n}(\tau)\right) \mid \begin{array}{l}
\tau=t \\
\tau=0
\end{array} \\
& s_{2} \leq K t \\
& s_{3} \leq K t .
\end{aligned}
$$

\section{(c) Existence of the solution}

Based on the estimates of the Galerkin approximations $v^{n}$ independent of $n \in \mathbb{N}$ we can prove a first main result:

Theorem 5.7. Let $v_{0} \in H_{2}(\Omega) \cap \mathcal{H}_{1}(\Omega)$ and let $X, Y$ be measure conserving homomorphisms in $\bar{\Omega}$. Then there is a uniquely determined function $v \in C\left([0, \varepsilon], H_{2}(\Omega) \cap \mathcal{H}_{1}(\Omega)\right)$ with $\partial_{t} v \in C\left([0, \varepsilon], \mathcal{H}_{0}(\Omega)\right)$ and a uniquely determined function $\nabla p \in C\left([0, \varepsilon], L_{2}(\Omega)\right)$ as a solution of the equations $\left(N_{\varepsilon}^{0}\right)$. The function $v(t)$ satisfies for all $t \in[0, \varepsilon]$ the energy equation

$$
\|v(t)\|^{2}+2 \nu \int_{0}^{t}\|\nabla v(\tau)\|^{2} d \tau=\left\|v_{0}\right\|^{2}
$$

and the estimates

$$
\begin{gathered}
\|\nabla v(t)\|^{2}+\nu \int_{0}^{t}\|P \Delta v(\tau)\|^{2} d \tau \leq\left\|\nabla v_{0}\right\|^{2}+c_{\nu, \varepsilon}\left\|v_{0}\right\|^{2} t \\
\left\|\partial_{t} v(t)\right\|^{2}+\nu \int_{0}^{t}\left\|\nabla \partial_{\tau} v(\tau)\right\|^{2} d \tau \leq\left\|\partial_{t} v(0)\right\|^{2}+c_{\Omega, \nu, \varepsilon}\left\|v_{0}\right\|^{2} t .
\end{gathered}
$$


Moreover, for all $t \in[\alpha, \varepsilon], 0<\alpha<\varepsilon$, the estimates (5.18), (5.19) and (5.20)also hold true, where here the constants on the right hand sides, in particular, depend on $\alpha$.

Proof: Let $v^{n}, n \in \mathbb{N}$ the Galerkin approximation defined by (5.2). Then we obtain from the estimates of Lemma 5.5 using the theorem of Arzela and Ascoli that for every $k \in \mathbb{N}_{0}$ the sequence $\left(\partial_{t}^{k} v^{n}\right)_{n}$ is relatively compact in $C\left([\alpha, \varepsilon], \mathcal{H}_{1}(\Omega)\right), 0<\alpha<\varepsilon$.

Using Lemma 5.4, in the case $k=0$ even $\alpha=0$ is allowed. By subsequently $(k=0,1, \ldots)$ extracting subsequences we finally obtain a subsequence, denoted by $\left(v^{\tilde{n}}\right)_{\tilde{n}}$, and a function $\tilde{v} \in C\left([0, \varepsilon], \mathcal{H}_{1}(\Omega)\right)$ with $\partial_{t}^{k} \tilde{v} \in C\left([\alpha, \varepsilon], \mathcal{H}_{1}(\Omega)\right)$ for all $k \in \mathbb{N}$, satisfying

$$
\sup _{[0, \varepsilon]}\left\|\nabla v^{\tilde{n}}(t)-\nabla \tilde{v}(t)\right\| \stackrel{\tilde{n} \rightarrow \infty}{\longrightarrow} 0
$$

and

$$
\sup _{[\alpha, \varepsilon]}\left\|\nabla \partial_{t}^{k} v^{\tilde{n}}(t)-\nabla \partial_{t}^{k} \tilde{v}(t)\right\| \stackrel{\tilde{n} \rightarrow \infty}{\longrightarrow} 0
$$

for every $\alpha$ with $0<\alpha<\varepsilon$ and every $k \in \mathbb{N}$.

Using the properties of weakly convergent subsequences in Sobolev spaces we find that every bound being independent of $n$ of the functions $v^{n}(t)$ from Lemma 5.4 and Lemma 5.5 also holds true for the limit function $\tilde{v}(t)$, as well as the estimate (5.21). This means that $\tilde{v}$ is a solution of the equations $\left(N_{\varepsilon}^{0}\right)$ such that the equation

$$
\partial_{\tau} \tilde{v}-\nu P \Delta \tilde{v}=-P Z_{\varepsilon}^{0} \tilde{v}
$$

is satisfied as identity in $L_{2}\left(0, t, \mathcal{H}_{0}(\Omega)\right)$ for all $0<t<\varepsilon$ with

$$
\lim _{t \searrow 0}\left\|\nabla \tilde{v}(t)-\nabla v_{0}\right\|=0 .
$$

Due to the measure conserving property of the mappings $X, Y$ we find $\left(Z_{\varepsilon}^{0} \tilde{v}(t), \tilde{v}(t)\right)=0$ for all $t \in[0, \varepsilon]$, and the energy equation follows from (5.25).

Moreover, the existence of a uniquely determined pressure gradient $\nabla \tilde{p}(t)$ is obtained from the projection theorem (compare [13]).

All the properties derived for the function $\tilde{v}$ are also valid for any other accumulation point, which is obtained by extracting some other different subsequences. Since, due to the linearity of the equations $\left(N_{\varepsilon}^{0}\right)$, such solutions are uniquely determined there is only one accumulation point $v=\tilde{v}$, and the whole sequence of Galerkin approximations $v^{n}$ converges to $v$ in the corresponding spaces.

Thus for the proof of the theorem it remains to show that the regularity $v \in C\left([0, \varepsilon], H_{2}(\Omega)\right)$ holds true. Using the estimate of Cattabriga ([2]), for any function $w \in H_{2}(\Omega) \cap \mathcal{H}_{1}(\Omega)(\partial \Omega \in$ $C^{2}$ ) we have

$$
\|w\|_{2} \leq c_{\Omega}\|P \Delta w\|
$$


From (5.19) we obtain by the Cauchy-Schwarz inequality the strong $H_{2}$-continuity of the function $t \rightarrow v(t)$ for every $t>0$ :

$$
\|P \Delta(t+h)-P \Delta v(t)\|^{2} \leq h \int_{t}^{t+h}\left\|P \Delta \partial_{\tau} v(\tau)\right\|^{2} d \tau \leq h K_{2} \quad(h>0) .
$$

To prove the strong $L_{2}$-continuity of the function $t \rightarrow P \Delta v(t)$ at time $t=0$ we firstly show the weak $L_{2}$-continuity of this function at $t=0$ as in $([3])$.

From here we quote (see [11])

$$
\left\|P \Delta v_{0}\right\| \leq \liminf _{t \searrow 0}\|P \Delta v(t)\|
$$

On the other hand, from (5.21) we obtain

$$
\limsup _{t \searrow 0}\|P \Delta v(t)\| \leq\left\|P \Delta v_{0}\right\|
$$

due to $\left\|P \Delta v^{n}(0)\right\| \leq\left\|P \Delta v_{0}\right\|$ for $n \in \mathbb{N}$ and the strong $L_{2}$-continuity of the functions $t \rightarrow v(t, Y)$ and $t \rightarrow v\left(t, Y^{-1}\right)$.

From the continuity of the function $t \rightarrow\|P \Delta v(t)\|$ in $t=0$ due to (5.28) and (5.29) finally it follows $v \in C\left([0, \varepsilon], H_{2}(\Omega)\right)$, and the theorem is proved.

\section{Solutions compatible at initial time $t=0$}

In Section 4 we proved the existence and uniqueness of a solution $v \in C\left([0, \varepsilon], H_{2}(\Omega) \cap \mathcal{H}_{1}(\Omega)\right)$ with $\partial_{t} v \in C\left([0, \varepsilon], \mathcal{H}_{0}(\Omega)\right)$ of the problem $\left(N_{\varepsilon}^{0}\right)$.

In this case we assumed the regularity $v_{0} \in H_{2}(\Omega) \cap \mathcal{H}_{1}(\Omega)$ for the initial value and, in addition, the mappings $X, Y$ to be measure conserving homomorphisms in $\bar{\Omega}$.

Next we want to show that the solution $v$ together with its time derivatives $\partial_{t}^{k} v(k \in \mathbb{N})$ of general order is contained in $C\left([0, \varepsilon], H_{m}(\Omega) \cap \mathcal{H}_{1}(\Omega)\right)$ for $m \in\{3,4\}$, if, in addition, the mappings $X, Y$ are $C^{m-2}$-diffeomorphisms in $\bar{\Omega}$.

This statement follows from the estimate of Cattabriga ([2]), which can be applied in $\Omega$ for $t>0$ and $k \in \mathbb{N}_{0}$ to the system

$$
P \Delta \partial_{t}^{k} v(t)=\frac{1}{\nu} P\left\{\partial_{t}^{k+1} v(t)+\partial_{t}^{k} Z_{\varepsilon}^{0} v(t)\right\}=: R_{k}(t) .
$$


After that we shall investigate a necessary and sufficient condition for $v \in C\left([0, \varepsilon], H_{m}(\Omega)\right.$ $\left.\cap \mathcal{H}_{1}(\Omega)\right)$ with $m \in\{3,4\}$. In particular, we shall see that the assumption $v_{0} \in H_{m}(\Omega) \cap$ $\mathcal{H}_{1}(\Omega)$ without further requirements is not sufficient for the strong $H_{m}$-continuity of the solution $t \rightarrow v(t)$ of $\left(N_{\varepsilon}^{0}\right)$ uniformly in time (compare [14]).

\section{(a) $H_{4}$-regularity for $t>0$}

The next lemma shows regularity properties of the solution $v(t)$ from Theorem 5.7 for $t>0$. Here no additional assumptions on the initial value $v_{0}$ are necessary.

Lemma 6.1. Let the assumptions of Theorem 5.7 be satisfied, and suppose, in addition, that the mappings in $\left(N_{\varepsilon}^{0}\right)$ satisfy $X, X^{-1}, Y, Y^{-1} \in C^{m-2}(\bar{\Omega})$ with $m \in\{3,4\}$. Then for the solution $v$ of $\left(N_{\varepsilon}^{0}\right)$ constructed in Theorem 5.7 we find $\partial_{t}^{k} v \in C\left((0, \varepsilon], H_{m}(\Omega) \cap \mathcal{H}_{1}(\Omega)\right)$ for all $k \in \mathbb{N}_{0}$.

Proof: Let $t \in(0, \varepsilon], k \in \mathbb{N}_{0}$, and $m \in\{3,4\}$ be given. Using (6.1) and the estimate of Cattabriga we have to verify $R_{k}(t) \in H_{m-2}(\Omega)$. For the term $\partial_{t}^{k+1} v(t)$ this follows from (5.20) using (5.27).

To prove $\partial_{t}^{k} Z_{\varepsilon}^{0} v(t) \in H_{m-2}(\Omega)$ we use the representation (5.7) of $\partial_{t}^{k} Z_{\varepsilon}^{0} v(t)$ to derive suitable bounds for the terms $\left\|\partial_{t}^{k} Z_{\varepsilon}^{0} v(t)\right\|^{2},\left\|\nabla\left(\partial_{t}^{k} Z_{\varepsilon}^{0} v(t)\right)\right\|^{2}$, and $\left\|\nabla^{2}\left(\partial_{t}^{k} Z_{\varepsilon}^{0} v(t)\right)\right\|^{2}$ her.

Due to the measure conserving property of $X, Y$ and (5.18) it follows

$$
\left\|\partial_{t}^{k} Z_{\varepsilon}^{0} v(t)\right\|^{2} \leq c_{k, \varepsilon}\left\{\left\|\partial_{t}^{k} v(t)\right\|^{2}+\left\|\partial_{t}^{k-1} v(t)\right\|^{2}\right\} \leq \tilde{K}_{1}
$$

where in the case $k=0$ the norm $\left\|\partial_{t}^{k-1} \cdot\right\|$ can be neglected.

To estimate the term $\left\|\nabla\left(\partial_{t}^{k} Z_{\varepsilon}^{0} v(t)\right)\right\|^{2}$, let $a$ denote some constant satisfying $\|\nabla g\|_{\infty} \leq a$ for every mapping $g \in \mathcal{M}=\left\{X, X^{-1}, Y, Y^{-1}\right\}$. Because of

$$
\left\|\nabla\left(\partial_{t}^{k} v(t, g)\right)\right\| \leq\left\|\nabla \partial_{t}^{k} v(t)\right\| \cdot\|\nabla g\|_{\infty}
$$

from (5.19) we find

$$
\left\|\nabla\left(\partial_{t}^{k} Z_{\varepsilon}^{0} v(t)\right)\right\|^{2} \leq c_{k, \varepsilon} a^{2}\left\{\left\|\nabla \partial_{t}^{k} v(t)\right\|^{2}+\left\|\nabla \partial_{t}^{k-1} v(t)\right\|^{2}\right\} \leq a^{2} \tilde{K}_{2} .
$$

Here as above, for $k=0$ the norm $\left\|\nabla \partial_{t}^{k-1} \cdot\right\|$ can be neglected.

Now let $m=4$ and denote by $b$ some constant satisfying $\left\|\nabla^{2} g\right\|_{\infty} \leq b$ for $g \in \mathcal{M}$, in addition. From

$$
\begin{aligned}
\left\|\nabla^{2}\left(\partial_{t}^{k} v(t)\right)\right\| & =\left\|\nabla_{g}^{2}\left(\partial_{t}^{k} v(t, g)\right) \cdot\left(\nabla_{g}\right)^{2}+\nabla_{g} \partial_{t}^{k} v(t, g) \cdot \nabla^{2} g\right\| \\
& \leq C_{\Omega} a^{2}\left\|P \Delta \partial_{t}^{k} v(t)\right\|+b\left\|\nabla \partial_{t}^{k} v(t)\right\|
\end{aligned}
$$


using (5.27), we find by (5.19) and (5.20) the estimate

$$
\begin{aligned}
\left\|\nabla^{2}\left(\partial_{t}^{k} Z_{\varepsilon}^{0} v(t)\right)\right\|^{2} \leq & C_{k, \varepsilon, \Omega}\left\{a^{4}\left\{\left\|P \Delta \partial_{t}^{k} v(t)\right\|^{2}+\left\|P \Delta \partial_{t}^{k-1} v(t)\right\|^{2}\right\}\right. \\
& \left.+b^{2}\left\{\left\|\nabla \partial_{t}^{k} v(t)\right\|^{2}+\left\|\nabla \partial_{t}^{k-1} v(t)\right\|^{2}\right\}\right\} \\
\leq & a^{4} \tilde{K}_{3}+b^{2} \tilde{K}_{2},
\end{aligned}
$$

where for $k=0$ the norms $\left\|\cdot \partial_{t}^{k-1} \cdot\right\|$ can be neglected.

Collecting the estimates (6.2), (6.3), and (6.4) we obtain $\partial_{t}^{k} v(t) \in H_{m}(\Omega) \cap \mathcal{H}_{1}(\Omega)$ for $m \in\{3,4\}, k \in \mathbb{N}_{0}$, and $t \in(0, \varepsilon]$, and the asserted continuity for $t>0$ follows from

$$
\left\|\partial^{\alpha} \partial_{t}^{k}(v(t+h)-v(t))\right\|^{2} \leq\left|h \cdot \int_{t}^{t+h}\left\|\partial^{\alpha} \partial_{\tau}^{k} v(\tau)\right\|^{2} d \tau\right| \quad(h \in \mathbb{R})
$$

for any spatial derivative of order $|\alpha| \leq m$.

\section{(b) The compatibility condition at time $t=0$}

The regularity property $v \in C\left(\left[0, T^{*}\right], H_{3}(\Omega) \cap \mathcal{H}_{1}(\Omega)\right)$ of a strong solution $v$ of the NavierStokes initial value problem $\left(N_{0}\right)$ - here $\left[0, T^{*}\right]$ denotes the maximum existence interval of the strong solution - leads to an over-determined Neumann problem for the pressure $p_{0}=p(0)$ at time $t=0$ (initial pressure). Due to its nonlocal character it is in general not checkable for given data (see [4] and [9]).

For the construction of solutions of the Navier-Stokes system $\left(N_{0}\right)$ with such a high degree of regularity it is necessary that some compatibility conditions are satisfied by the velocity field on the parabolic boundary (see [14]). Similar conditions for the corresponding linear problem can be find in [14] and [7]. The compatibility condition for the problem $\left(N_{\varepsilon}^{0}\right)$ is formulated in the next theorem. It can be satisfied - as shown in the next section - due to a special construction of the initial values (compare Remark 3.6).

Theorem 6.2. Let the assumptions of Lemma 6.1 be satisfied and assume, in addition, $v_{0} \in H_{m}(\Omega) \cap \mathcal{H}_{1}(\Omega)$ for $m \in\{3,4\}$. Let $v$ denote the solution of Problem $\left(N_{\varepsilon}^{0}\right)$ from Theorem 5.7. Then it holds $v \in C\left([0, \varepsilon], H_{m}(\Omega) \cap \mathcal{H}_{1}(\Omega)\right)$ if and only if

$$
\nu P \Delta v_{0_{\partial \Omega}}-\frac{1}{2 \varepsilon} P\left\{v_{0} \circ Y-v_{0} \circ Y^{-1}\right\}_{\left.\right|_{\partial \Omega}}=0 .
$$

Moreover, from (6.5) it follows $\partial_{t} v \in C\left([0, \varepsilon], H_{m-2}(\Omega) \cap \mathcal{H}_{1}(\Omega)\right)$, as well as, in addition, $\partial_{t}^{2} v \in C\left([0, \varepsilon], \mathcal{H}_{0}(\Omega)\right)$ for $m=4$.

Remark 6.3. Using the projection $P$ in the equations $\left(N_{\varepsilon}^{0}\right)$, we obtain at time $t=0$

$$
\nu P \Delta v_{0}-\frac{1}{2 \varepsilon} P\left\{v_{0} \circ Y-v_{0} \circ Y^{-1}\right\}=\partial_{t} v(0) .
$$

Hence for $\partial_{t} v(0) \in \mathcal{H}_{1}(\Omega)$ the condition (6.5) is satisfied (compare (4.3) and Lemma 4.1). 
Remark 6.4. The proof of the above theorem uses some statements about the solution $w=$ $\left(w_{1}, w_{2}, w_{3}\right)$ of the non-stationary Stokes initial boundary value problem

$$
\begin{aligned}
\partial_{t} w-\nu P \Delta w & =g \quad(t, x) \in(0, T] \times \Omega \\
w_{\left.\right|_{t=0}} & =w_{0} .
\end{aligned}
$$

Here we recall:

(a) For $w_{0} \in \mathcal{H}_{1}(\Omega)$ and $g \in L_{2}\left(0, T, \mathcal{H}_{0}(\Omega)\right)$ there exists a uniquely determined solution $w$ of Problem (6.7), and it holds $w \in C([0, T]), \mathcal{H}_{1}(\Omega)$ with $\partial_{t} w \in L_{2}\left(0, T, \mathcal{H}_{0}(\Omega)\right.$ ) (see [7]).

(b) If, in addition, $w_{0} \in H_{2}(\Omega)$ and $\partial_{t} g \in L_{2}\left(0, T, \mathcal{H}_{0}(\Omega)\right)$, then, in addition, we even find $w \in C\left([0, T], H_{2}(\Omega) \cap \mathcal{H}_{1}(\Omega)\right)$ with $\partial_{t} w \in C\left([0, T], \mathcal{H}_{0}(\Omega)\right)$ (see $\left.[7]\right)$.

Proof of Theorem 6.2: Let $v \in C\left([0, \varepsilon], H_{3}(\Omega) \cap \mathcal{H}_{1}(\Omega)\right)$ be a solution of the problem $\left(N_{\varepsilon}^{0}\right)$. Then it follows $v(0) \in \mathcal{H}_{1}(\Omega)$ and $\partial_{t} v(0) \in H_{1}(\Omega)$. Since $\mathcal{H}_{1}(\Omega)$ is a closed subspace of $H_{1}(\Omega)$ we find $\partial_{t} v(0) \in \mathcal{H}_{1}(\Omega)$, and (6.5) follows from Remark 6.3.

Let us assume now that the equation (6.5) holds. Differentiate the equation $\left(N_{\varepsilon}^{0}\right)$ with respect to $t$ and consider the resulting equations as a problem of the type (6.7) for $w=\partial_{t} v$ with $T=\varepsilon, g=-P \partial_{t} Z_{\varepsilon}^{0} v$ and $w_{0}$ given by (6.6).

For this problem the assumptions in Remark 6.4 (a) are satisfied: Due to $\partial_{t} v \in L_{2}\left(0, \varepsilon, \mathcal{H}_{0}(\Omega)\right)$ using (5.25) it follows $g \in L_{2}\left(0, \varepsilon, \mathcal{H}_{0}(\Omega)\right)$, and since $v_{0} \in H_{m}(\Omega) \cap \mathcal{H}_{1}(\Omega)$ due to the assumption we find $w_{0}=\partial_{t} v(0) \in H_{m-2}(\Omega) \cap \mathcal{H}_{0}(\Omega)$, hence using (6.5) we have $w_{0} \in \mathcal{H}_{1}(\Omega)$. Therefore we quote $w=\partial_{t} v \in C\left([0, \varepsilon], \mathcal{H}_{1}(\Omega)\right)$ with $\partial_{t} w=\partial_{t}^{2} v \in L_{2}\left(0, \varepsilon, \mathcal{H}_{0}(\Omega)\right)$. This implies $\partial_{t} g \in L_{2}\left(0, \varepsilon, \mathcal{H}_{0}(\Omega)\right)$, and in the case $m=4$ also $w=\partial_{t} v \in\left([0, \varepsilon], H_{m-2}(\Omega) \cap \mathcal{H}_{1}(\Omega)\right)$ with $\partial_{t} w=\partial_{t}^{2} v \in C\left([0, \varepsilon], \mathcal{H}_{0}(\Omega)\right)$, using Remark $6.4(\mathrm{~b})$.

Due to

$$
\nu P \Delta v=\partial_{t} v+P Z_{\varepsilon}^{0} v \in C\left([0, \varepsilon], H_{m-2}(\Omega) \cap \mathcal{H}_{0}(\Omega)\right)
$$

we finally obtain $v \in C\left([0, \varepsilon], H_{m}(\Omega) \cap \mathcal{H}_{1}(\Omega)\right)$ with help of Cattabriga's estimate.

\section{Global solutions}

In the first theorem of this section we prove the existence of a solution $v$ to the problem $\left(\tilde{N}_{\varepsilon}\right)$ formulated in $\S 2$ with $v \in C\left([0, T], H_{m}(\Omega) \cap \mathcal{H}_{1}(\Omega)\right)$ for $m \in\{3,4\}$.

This high degree of regularity can be proved since all compatibility conditions can be satisfied: At time $t_{0}=0$ we use a special construction of the initial values as shown in Section 3 (we 
prescribe $\left.\partial_{t} v(0)=f \in \mathcal{H}_{1}(\Omega)\right)$ as initial acceleration $)$, and at time $t_{k}>0(k=1, \ldots, N-1)$ we use the continuity of the mappings $Z_{\varepsilon} v(\cdot, x)$ for $x \in \bar{\Omega}$ (the function $\partial_{t} v\left(t_{k}\right)$ as final value of the $k$-th partial problem coincides with the initial value of the next partial problem).

Moreover we show that the solution is uniquely determined if the initial values are constructed as indicated in Section 3, and that the solution satisfies the energy equation.

Theorem 7.1. Let $f \in H_{m-2}(\Omega) \cap \mathcal{H}_{1}(\Omega)$ for $m \in\{3,4\}$. With $f$ and some function $\bar{v} \in C\left([-T, 0], H_{m}(\Omega) \cap \mathcal{H}_{2}(\Omega)\right)$ let the initial values for the problem $\left(\tilde{N}_{\varepsilon}\right)$ be constructed uniquely as indicated in Section 3. Then there exists a uniquely determined function $v \in$ $C\left([0, T], H_{m}(\Omega) \cap \mathcal{H}_{1}(\Omega)\right)$ with $\partial_{t} v \in C\left([0, T], H_{m-2}(\Omega) \cap \mathcal{H}_{1}(\Omega)\right)$ and a uniquely determined function $\nabla p \in C\left([0, T], H_{m-2}(\Omega)\right)$ as solution of the problem $\left(\tilde{N}_{\varepsilon}\right)$.

Proof: Using $v$ and $\bar{v}$, first we construct the initial mappings $Y:=X_{t_{-1}, t_{-2}}, Y^{-1}:=X_{t_{-2}, t_{-1}}$, $v_{0}, X:=X_{t_{0}, t_{-1}}$ and $X^{-1}:=X_{t_{-1}, t_{0}}$ as done in Section 3 .

Then, with these functions as given data, we consider the problem $\left(\tilde{N}_{\varepsilon}\right)$ restricted to the interval $[0, \varepsilon]$ as a problem of the type $\left(N_{\varepsilon}^{0}\right)$.

Due to Section 1 and Lemma 4.1 all the assumptions of Theorem 6.2 are fulfilled, and, following Remark 6.3, due to $f \in \mathcal{H}_{1}(\Omega)$ also the compatibility condition (6.5) is satisfied. It follows that there is a uniquely determined function $v^{0}$ as solution of $\left(N_{\varepsilon}^{0}\right)$ (and thus of $\left(\tilde{N}_{\varepsilon}\right)$, restricted to $\left.[0, \varepsilon]\right)$ with the following regularity properties:

$v^{0} \in C\left([0, \varepsilon], H_{m}(\Omega) \cap \mathcal{H}_{1}(\Omega)\right)$ with $\partial_{t} v \in C\left([0, \varepsilon], H_{m-2}(\Omega) \cap \mathcal{H}_{1}(\Omega)\right)$ and for $m=4$ also $\partial_{t}^{2} v \in C\left([0, \varepsilon], \mathcal{H}_{0}(\Omega)\right)$; moreover we have $v^{0}(0)=v_{0}$ and $\partial_{t} v^{0}(0)=f$.

This solution procedure for the problem $\left(\tilde{N}_{\varepsilon}\right)$ can be repeated: First we construct the mappings $X_{t_{1}, t_{0}}$ and $X_{t_{0}, t_{1}}$ from the function $v^{0}$, since they are needed in the next subinterval $[\varepsilon, 2 \varepsilon]$ (according to Lemma 2.1, this is possible due to $v^{0} \in C\left([0, \varepsilon], H_{m}(\Omega) \cap \mathcal{H}_{1}(\Omega)\right)$ ), and then we consider the problem $\left(\tilde{N}_{\varepsilon}\right)$ restricted to the subinterval $[\varepsilon, 2 \varepsilon]$ as problem

$$
\begin{aligned}
\partial_{t} v-\nu \Delta v+\nabla p & =-Z_{\varepsilon}^{1} v \\
\nabla \cdot v & =0 \\
v_{\left.\right|_{\partial \Omega}} & =0 \\
v_{\left.\right|_{t=\varepsilon}} & =v^{0}(\varepsilon),
\end{aligned}
$$$$
(t, x) \in(\varepsilon, 2 \varepsilon] \times \Omega
$$

where $Z_{\varepsilon}^{1} v$ is defined by 3.5 .

We denote the solution of this problem by $v^{1}$. This solution has the same properties as the solution $v^{0}$ of the problem $\left(N_{\varepsilon}^{0}\right)$ : Due to $v^{1}(\varepsilon)=v^{0}(\varepsilon), Z_{\varepsilon}^{1} v^{1}(\varepsilon)=Z_{\varepsilon}^{0} v^{0}(\varepsilon)$ and the unique solvability of Problem (4.3) we find $\partial_{t} v^{1}(\varepsilon)=\partial_{t} v^{0}(\varepsilon) \in H_{m-2}(\Omega) \cap \mathcal{H}_{1}(\Omega)$. Hence the compatibility condition corresponding to (6.5) for the problem $\left(N_{\varepsilon}^{1}\right)$ at time $t_{1}=\varepsilon$ is 
satisfied, too. It follows that the solution $v^{1}$ of this problem is uniquely determined, and we have $v^{1} \in C\left([\varepsilon, 2 \varepsilon], H_{m}(\Omega) \cap \mathcal{H}_{1}(\Omega)\right)$ with $\partial_{t} v^{1} \in C\left([\varepsilon, 2 \varepsilon], H_{m-2}(\Omega) \cap \mathcal{H}_{1}(\Omega)\right)$, and for $m=4$ also $\partial_{t}^{2} v^{1} \in C\left([\varepsilon, 2 \varepsilon], \mathcal{H}_{0}(\Omega)\right)$. Moreover, we find $v^{1}(\varepsilon)=v^{0}(\varepsilon)$ and $\partial_{t} v^{1}(\varepsilon)=\partial_{t} v^{0}(\varepsilon)$, but not in general $\partial_{t}^{2} v^{1}(\varepsilon)=\partial_{t}^{2} v^{0}(\varepsilon)(m=4)$.

By repeating this solution procedure we finally obtain functions $v^{k}$ defined on subsequent subintervals $\left[t_{k}, t_{k-1}\right]$, which we can put together to a uniquely determined solution $v$ of the problem $\left(\tilde{N}_{\varepsilon}\right)$ in such a way that all properties asserted in Theorem 7.1 are satisfied.

To investigate convergence in the next section we need the regularized problem $\left(N_{\varepsilon}\right)$, , which is obtained from $\left(\tilde{N}_{\varepsilon}\right)$ by changing the initial condition: Let $T>0, N \in \mathbb{N}(N \geq 2), \varepsilon:=\frac{T}{N}$ and $t_{k}=k \cdot \varepsilon$ for $k=0, \pm 1, \ldots, \pm N$ as in Problem $\left(\tilde{N}_{\varepsilon}\right)$.

Construct a velocity field $v=\left(v_{1}, v_{2}, v_{3}\right)$ and some pressure function $p$ as a solution of the problem

$$
\begin{array}{rlr}
\partial_{t} v-\nu \Delta v+\nabla p & =-Z_{\varepsilon} v & \\
\nabla \cdot v & =0 & \\
v_{\mid \partial \Omega} & =0 & \\
v_{\mid t=0} & =v_{0}(\varepsilon), &
\end{array}
$$

where $Z_{\varepsilon} v$ is defined as in 3.5 .

Theorem 7.1 leads to a statement about the solvability of the problem $\left(N_{\varepsilon}\right)$ :

Corollary 7.2. Let the assumptions of Theorem 7.1 be satisfied, and let the initial data for the problem $\left(N_{\varepsilon}\right)$ coincide with the initial data of problem $\left(\tilde{N}_{\varepsilon}\right)$ from Theorem 7.1 . Then, given $f \in H_{m-2}(\Omega) \cap \mathcal{H}_{1}(\Omega)$ for $m \in\{3,4\}$ as initial value in Problem $\left(\tilde{N}_{\varepsilon}\right)$, there is a uniquely determined function $v_{0} \in H_{m}(\Omega) \cap \mathcal{H}_{1}(\Omega)$ as the solution of Problem 4.3. Using this function $v_{0}$ as initial value the problem $\left(N_{\varepsilon}\right)$ is uniquely solvable.

Its solution $v$ coincides with the solution of the problem $\left(\tilde{N}_{\varepsilon}\right)$ from Theorem 7.1 and satisfies in $t \in[0, T]$ the energy equation

$$
\|v(t)\|^{2}+2 \nu \int_{0}^{t}\|\nabla v(\tau)\|^{2} d \tau=\left\|v_{0}\right\|^{2} .
$$

Proof: We only have to show (7.1). This follows from (5.22), since for $t \in\left[t_{k}, t_{k+1}\right]$ and 
$k=0,1, \ldots, N-1$ we find

$$
\begin{aligned}
\|v(t)\|^{2}-\left\|v\left(t_{k}\right)\right\|^{2} & +2 \nu \int_{t_{k}}^{t}\|\nabla v(\tau)\|^{2} d \tau+\sum_{j=1}^{k}\left\{\left\|v\left(t_{j}\right)\right\|^{2}-\left\|v\left(t_{j-1}\right)\right\|^{2}\right. \\
& \left.+2 \nu \int_{t_{j-1}}^{t_{j}}\|\nabla v(\tau)\|^{2} d \tau\right\}=0 .
\end{aligned}
$$

The properties

$$
v \in C\left([0, T], H_{4}(\Omega) \cap \mathcal{H}_{1}(\Omega)\right), \partial_{t} v \in C\left([0, T], H_{2}(\Omega) \cap \mathcal{H}_{1}(\Omega)\right)
$$

for the solution $v$ of the problem $\left(\tilde{N}_{\varepsilon}\right)$ and $\left(N_{\varepsilon}\right)$, respectively, represent the highest degree of regularity - formulated in standard Sobolev spaces of integer order - which is possible without using additional (coupled) compatibility conditions at time $t=0$ (see [14]). These properties imply, in particular, that the solution $v$ is continuously differentiable one time with respect to $t$ and two times with respect to $x$ in the open cylinder $\Omega_{T}:=(0, T) \times \Omega$, and the corresponding derivatives can be continuously extended to the parabolic boundary of $\Omega_{T}$. In this sense the function $v$ is a classical solution of the problem $\left(\tilde{N}_{\varepsilon}\right)$, and we finish the regularity investigation of this problem at this stage.

\section{Global Convergence to a weak solution of $\left(N_{0}\right)$}

In this section we suppose that the initial data for the problems $\left(\tilde{N}_{\varepsilon}\right)$ and $\left(N_{\varepsilon}\right)$ are constructed as in Section 3, and that the initial value $v_{0}$ given in the problem $\left(N_{\varepsilon}\right)$ has been determined as the solution of the problem (4.3) from the initial value $f$ of the problem $\left(\tilde{N}_{\varepsilon}\right)$.

Under these assumptions we showed in the last Section 6 , that the problems $\left(\tilde{N}_{\varepsilon}\right)$ and $\left(N_{\varepsilon}\right)$ are globally uniquely solvable and that their solutions coincide.

In the following we investigate the behavior of the solution of $\left(N_{\varepsilon}\right)$, if for fixed $T>0$ the step size $\varepsilon=\frac{T}{N}$ for $N \rightarrow \infty$ tends to zero.

To do so, we set $\varepsilon_{N}:=\frac{T}{N}$ for $2 \leq N \in \mathbb{N}$ and denote the solution of the problem $\left(N_{\varepsilon_{N}}\right)$ from Corollary 7.2 by $v^{N}$. The corresponding initial value, depending on $\varepsilon_{N}$ as well, is denoted by $v_{0}^{N}$. Moreover, let $\tilde{v}^{N}$ be the function defined by (4.4), necessary for the construction of the initial values. Here we start with some given function $\bar{v}^{N}$ for $N=2$ as in Section 3, and then we choose $\bar{v}^{N}:=\tilde{v}^{N-1}$ for all $N \geq 3$ : 
The reason for this choice of the functions $\bar{v}^{N}$ will be explained in the next section. All statements of this section also remain true if we simply choose $\bar{v}^{N}:=\bar{v}^{2}=: \bar{v}$.

The definition of a weak solution of the Navier-Stokes problem $\left(N_{0}\right)$ in the sense of Hopf (compare [5], [13], [10], [7]) is given now:

Definition 8.1. Let $v_{0} \in \mathcal{H}_{0}(\Omega)$. A function $v \in L_{2}\left(0, T, \mathcal{H}_{1}(\Omega)\right) \cap L_{\infty}\left(0, T, \mathcal{H}_{0}(\Omega)\right)$ is a weak solution of the problem $\left(N_{0}\right)$ with initial value $v_{0}$, if

$$
\begin{gathered}
v:[0, T] \longrightarrow \mathcal{H}_{0}(\Omega) \quad \text { is weakly continuous } \\
\lim _{t \rightarrow 0}\left\|v(t)-v_{0}\right\|=0
\end{gathered}
$$

and for all test functions $\phi \in C_{0, \sigma}^{\infty}\left(\Omega_{T}\right)$ we have

$$
\int_{0}^{T}\left\{\left(v(t), \partial_{t} \phi(t)\right)-\nu(\nabla v(t), \nabla \phi(t))\right\} d t=-\int_{0}^{T}(v(t) \cdot \nabla \phi(t), v(t)) d t .
$$

Remark 8.2. It can be shown that every function $v \in L_{2}\left(0, T, \mathcal{H}_{1}(\Omega)\right)$ satisfying (8.2) and (8.3) already represents a weak solution of the problem $\left(N_{0}\right)$ with initial value $v_{0} \in \mathcal{H}_{0}(\Omega)$, according to Definition 8.1 (see [13]).

The following theorem states the main convergence result and shows that only the central total difference quotient leads to an energy conserving regularizing approximation for the convective term of the Navier-Stokes system (compare Remark 3.2).

Theorem 8.3. Let $T \in \mathbb{R}(T>0)$ and $N \in \mathbb{N}(N \geq 2)$. Setting $\varepsilon_{N}:=\frac{T}{N}$ let $v^{N}$ denote the uniquely determined solution of the problem $\left(N_{\varepsilon_{N}}\right)$ with initial value $v_{0}^{N}$ from Corollary 7.2. Then there is a convergent subsequence $\left(v^{N_{k}}\right)_{k}$ of the sequence $\left(v^{N}\right)_{N}$ of the solutions with limit function $v$ and a convergent subsequence $\left(v_{0}^{N_{k}}\right)_{k}$ of the sequence $\left(v_{0}^{N}\right)_{N}$ of the corresponding initial values with limit function $v_{0}$ such that $v$ is a weak solution of the Navier-Stokes problem $\left(N_{0}\right)$ with initial value $v_{0} \in \mathcal{H}_{0}(\Omega)$ and satisfies for $t \in[0, T]$ the energy inequality

$$
\|v(t)\|^{2}+2 \nu \int_{0}^{t}\|\nabla v(\tau)\|^{2} d \tau \leq\left\|v_{0}\right\|^{2}
$$

To prove Theorem 8.3 we need some estimates of the regularized solutions $v^{N}$ and their data independent of $N$. These estimates will be established in the next two lemmata.

Lemma 8.4. Let the assumptions of Theorem 8.3 be satisfied. Let $f$ denote the initial value of the problem $\left(\tilde{N}_{\varepsilon_{N}}\right)$, and let $\tilde{v}^{N}$ denote the function defined by (4.4) and constructed according to Section 3 from $\bar{v}$. Then the following estimates hold true independent of $N \in$ $\mathbb{N}(N \geq 2)$ :

$$
\left\|v_{0}^{N}\right\|^{2} \leq c_{\Omega, \nu}\|f\|^{2}
$$




$$
\left\|\nabla v_{0}^{N}\right\|^{2} \leq \tilde{c}_{\Omega, \nu}\|f\|^{2}
$$

as well as for all $t \in[0, T]$

$$
\left\|v^{N}(t)\right\|^{2}+2 \nu \int_{0}^{t}\left\|\nabla v^{N}(\tau)\right\|^{2} d \tau=\left\|v_{0}^{n}\right\|^{2} \leq c_{\Omega, \nu}\|f\|^{2},
$$

and for all $t \in[-T, 0]$

$$
\begin{gathered}
\left\|\tilde{v}^{N}(t)\right\| \leq \max \left\{\sup _{s \in[-T, 0]}\|\bar{v}(s)\|, c_{\Omega, \nu}\|f\|\right\}, \\
\left\|\nabla \tilde{v}^{N}(t)\right\| \leq \max \left\{\sup _{s \in[-T, 0]}\|\nabla \bar{v}(s)\|, \tilde{c}_{\Omega, \nu}\|f\|\right\}
\end{gathered}
$$

Proof: The estimates (8.5) and (8.6) follow using the inequality of Poincaré due to the measure conserving property of the mappings $X_{.,}$from (4.3). The estimate (8.7) is obtained from (7.1) and (8.8), and (8.9) follows from (4.4).

Lemma 8.5. Let $v^{N}$ denote the solution of the problem $\left(N_{\varepsilon_{N}}\right)$ from Theorem 8.3, and let $\mathcal{V}:=\left\{a_{i} \mid i \in \mathbb{N}\right\}$ be a complete orthonormal system in $\mathcal{H}_{0}(\Omega)$. Then for every $i \in \mathbb{N}$ we have the estimate

$$
\left|\left(Z_{\varepsilon_{N}} v^{N}(t), a_{i}\right)\right| \leq K_{i}
$$

where the constant $K_{i}$ does not depend on $N \in \mathbb{N}(N \geq 2)$ and not on $t \in[0, T]$.

Proof: Let $i, N \in \mathbb{N}(N \geq 2)$ and $t \in[0, T]$. For simplification we set $\varepsilon:=\varepsilon_{N}$ and obtain from the measure conserving property of the mappings $X_{., .}$analogously to partial integration the identity

$$
\left(Z_{\varepsilon} v^{N}(t), a_{i}\right)=-\left(Z_{\varepsilon} a_{i}, v^{N}(t)\right) .
$$

Here $Z_{\varepsilon} a_{i}$ for $t \in\left[t_{k}, t_{k+1}\right]$ and $k=0,1, \ldots, N-1$ is defined by $Z_{\varepsilon} a_{i}=\frac{t-t_{k}}{2 \varepsilon^{2}}\left\{a_{i} \circ X_{t_{k}, t_{k-1}}-a_{i} \circ X_{t_{k-1}, t_{k}}\right\}+\frac{t_{k+1}-t}{2 \varepsilon^{2}}\left\{a_{i} \circ X_{t_{k-1}, t_{k-2}}-a_{i} \circ X_{t_{k-2}, t_{k-1}}\right\}$.

By a well-known density argument we can choose $\mathcal{V} \subset C_{0, \sigma}^{\infty}(\Omega)$. Since the functions $X_{\text {., }}$ defined by (2.5) have been constructed from the solution of an initial value problem of type (2.4), we obtain, setting for abbreviation

$$
X_{k}:=X_{t_{k}, t_{k-1}} \quad(k=1,0, \ldots, N-1),
$$

for every $x \in \bar{\Omega}$ the following representation:

$$
\begin{aligned}
& a_{i} \circ X_{k}(x)-a_{i} \circ X_{k}^{-1}(x) \\
= & a_{i} \circ X_{k}(x)-a_{i}+a_{i}-a_{i} \circ X_{k}^{-1}(x)
\end{aligned}
$$




$$
\begin{aligned}
& =\int_{t_{k-1}}^{t_{k}}\left\{\partial_{\tau} X\left(\tau, t_{k-1}, x\right) \cdot \nabla_{X} a_{i}\left(X\left(\tau, t_{k-1}, x\right)\right)+\partial_{\tau} X\left(\tau, t_{k}, x\right) \cdot \nabla a_{i}\left(X\left(\tau, t_{k}, x\right)\right)\right\} d \tau \\
& =\int_{t_{k-1}}^{t_{k}}\left\{\left[v^{N}(\tau) \cdot \nabla a_{i}\right] \circ X\left(\tau, t_{k-1}, x\right)+\left[v^{N}(\tau) \cdot \nabla a_{i}\right] \circ X\left(\tau, t_{k}, x\right)\right\} d \tau .
\end{aligned}
$$

Here for $k \in\{-1,0\}$, due to the construction of the initial values according to Section 3 , the function $v^{N}$ has to be replaced by $\tilde{v}^{N}$. From

$$
\left|a_{i} \circ X_{k}(x)-a_{i} \circ X_{k}^{-1}(x)\right| \leq 2 \varepsilon \cdot \sup _{s_{1}, s_{2} \in[0, T]}\left|\left[v^{N}\left(s_{1}\right) \cdot \nabla a_{i}\right] \circ X\left(s_{1}, s_{2}, x\right)\right|
$$

for $k \in\{1,2, \ldots, N-1\}$ and from

$$
\left|a_{i} \circ X_{k}(x)-a_{i} \circ X_{k}^{-1}(x)\right| \leq 2 \varepsilon \cdot \sup _{s_{1}, s_{2} \in[-T, 0]}\left|\left[\tilde{v}^{N}\left(s_{1}\right) \cdot \nabla a_{i}\right] \circ X\left(s_{1}, s_{2}, x\right)\right|
$$

for $k \in\{-1,0\}$ it follows by (8.7), (8.8), and (8.12) that the estimate

$$
\left\|Z_{\varepsilon} a_{i}\right\| \leq \tilde{K}_{i}
$$

holds true. Here the constant does not depend on $\varepsilon$ and thus not on $N$. The assertion now follows using (8.7) from (8.11), and the lemma is proved.

Due to $(8.10)$ we obtain by projecting the problem $\left(N_{\varepsilon_{N}}\right)$ onto the subspace of $\mathcal{H}_{0}(\Omega)$ spanned by $a_{i} \in \mathcal{V}$ the estimate

$$
\left|\frac{d}{d t}\left(v^{N}(t), a_{i}\right)\right|=\left|\left(\partial_{t} v^{N}(t), a_{i}\right)\right| \leq \bar{K}_{i}
$$

where the constant $\bar{K}_{i}, i \in \mathbb{N}$, is independent of $N \in \mathbb{N}(N \geq 2)$ and $t \in[0, T]$. It depends only on $\Omega, \nu$, and the data $f, \bar{v}$, and the basis function $a_{i} \in \mathcal{V}$, where we may assume $\mathcal{V} \subset C_{0, \sigma}^{\infty}(\Omega)$ using a density argument, as already mentioned above.

As in [5] we obtain from (8.7) and (8.15)

Lemma 8.6. Let the assumptions of Theorem 8.3 be satisfied. Then there exists a weakly continuous function $v:[0, T] \rightarrow \mathcal{H}_{0}(\Omega)$ with

$$
v \in L_{2}\left(0, T, \mathcal{H}_{1}(\Omega)\right) \cap L_{\infty}\left(0, T, \mathcal{H}_{0}(\Omega)\right)
$$

and a subsequence $\left(v^{N_{k}}\right)_{k}$ of the sequence of the solutions of the problems $\left(N_{\varepsilon_{N}}\right)$ with the following properties: For $t \in[0, T]$ the sequence $\left(v^{N_{k}}(t)\right)_{k}$ converges weakly in $\mathcal{H}(\Omega)$ to the limit $v(t)$, and the sequence $\left(v^{N_{k}}\right)_{k}$ converges weakly to $v$ in $L_{2}\left(0, T, \mathcal{H}_{1}(\Omega)\right)$ and strongly in $L_{2}\left(0, T, \mathcal{H}_{0}(\Omega)\right)$.

The following theorem shows that the properties of the subsequence $\left(v^{N_{k}}\right)_{k}$ from Lemma 8.6 are already sufficient to proceed to the limit in the nonlinear convective term of the Navier-Stokes equations: 
Theorem 8.7. Let $\mathcal{V}:=\left\{a_{i} \mid i \in \mathbb{N}\right\}$ denote a complete orthonormal system in $\mathcal{H}_{0}(\Omega)$. Then for the convergent subsequence $\left(v^{N_{k}}\right)_{k}$ with limit function $v$ according to Lemma 8.6 for all $i \in \mathbb{N}$ the following identity holds true:

$$
\lim _{k \rightarrow \infty} \int_{0}^{T}\left(Z_{\varepsilon_{N_{k}}} a_{i}, v^{N_{k}}(t)\right) d t=\int_{0}^{T}\left(v(t) \cdot \nabla a_{i}, v(t)\right) d t .
$$

Proof: For simplification we set $\varepsilon:=\varepsilon_{N_{k}}, N:=N_{k}, a:=a_{i}, v^{N}(s):=\tilde{v}^{N}(s)$ for $s \in[-T, 0]$ and write (8.16) in the form

$$
\lim _{N \rightarrow \infty} \int_{0}^{T}\left\{\left(Z_{\varepsilon} a, v^{N}(t)\right)-(v(t) \cdot \nabla a, v(t))\right\} d t=0 .
$$

We prove (8.17) using a decomposition of the integrand $I^{N}(t)$ :

$$
I^{N}(t)=\left(Z_{\varepsilon} a, v^{N}(t)-v(t)\right)+\left(Z_{\varepsilon} a-v(t) \cdot \nabla a, v(t)\right)=: \tilde{S}_{1}^{N}(t)+\tilde{S}_{2}^{N}(t) .
$$

Due to (8.12) and the notation (8.13) we find

$$
\begin{aligned}
\tilde{S}_{2}^{n}(t)= & \frac{t-t_{k}}{2 \varepsilon}\left(\frac{1}{\varepsilon}\left\{a \circ X_{k}-a\right\}-v(t) \cdot \nabla a, v(t)\right)+ \\
& \frac{t-t_{k}}{2 \varepsilon}\left(\frac{1}{\varepsilon}\left\{a-a \circ X_{k}^{-1}\right\}-v(t) \cdot \nabla a, v(t)\right)+ \\
& \frac{t_{k+1}-t}{2 \varepsilon}\left(\frac{1}{\varepsilon}\left\{a \circ X_{k-1}-a\right\}-v(t) \cdot \nabla a, v(t)\right)+ \\
& \frac{t_{k+1}-t}{2 \varepsilon}\left(\frac{1}{\varepsilon}\left\{a-a \circ X_{k-1}^{-1}\right\}-v(t) \cdot \nabla a, v(t)\right) \\
= & \sum_{j=1}^{4} M_{j}^{N}(t) .
\end{aligned}
$$

The term $M_{1}^{N}(t)$ is decomposed again $\left(M_{2}^{N}(t)\right.$ to $M_{4}^{N}(t)$ analogously):

$$
\begin{aligned}
M_{1}^{N}(t) & =\frac{t-t_{k}}{2 \varepsilon}\left(\frac{1}{\varepsilon} \int_{t_{k-1}}^{t_{k}} \partial_{\tau} X\left(\tau, t_{k-1}, \cdot\right) \cdot \nabla a\left(X\left(\tau, t_{k-1}, \cdot\right)\right) d \tau-v(t) \cdot \nabla a, v(t)\right) \\
& =\frac{t-t_{k}}{2 \varepsilon^{2}} \int_{t_{k-1}}^{t_{k}}\left(\left[v^{N}(\tau) \cdot \nabla a\right] \circ X\left(\tau, t_{k-1}, \cdot\right)-v(t) \cdot \nabla a, v(t)\right) d \tau
\end{aligned}
$$




$$
\begin{aligned}
= & \frac{t-t_{k}}{2 \varepsilon^{2}} \int_{t_{k-1}}^{t_{k}}\left(\left[v^{N}(\tau)-v^{N}(t)\right] \cdot \nabla a, v(t) \circ X\left(t_{k-1}, \tau, \cdot\right)\right) d \tau+ \\
& \frac{t-t_{k}}{2 \varepsilon^{2}} \int_{t_{k-1}}^{t_{k}}\left(\left[v^{N}(t)-v(\tau)\right] \cdot \nabla a, v(t) \circ X\left(t_{k-1}, \tau, \cdot\right)\right) d \tau- \\
& \frac{t-t_{k}}{2 \varepsilon^{2}} \int_{t_{k-1}}^{t_{k}}\left(v(t) \cdot \nabla a, v(t)-v(t) \circ X\left(t_{k-1}, \tau, \cdot\right)\right) d \tau \\
= & \sum_{j=1}^{3} s_{j}^{N}(t) .
\end{aligned}
$$

The integrals of the summands $\tilde{S}_{1}^{N}(t)$ and $s_{1}^{N}(t), s_{2}^{N}(t), s_{3}^{N}(t)$ will be estimated now. In the following, all appearing constants $K_{1}, K_{2}, \ldots$ do not depend on $N$.

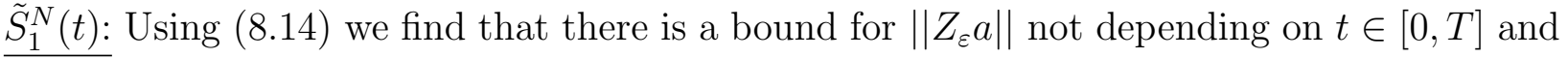
not depending on $\varepsilon$. This implies

$$
\left|\int_{0}^{T} \tilde{S}_{1}^{N}(t) d t\right| \leq \int_{0}^{T}\left\|Z_{\varepsilon} a\right\|\left\|v^{N}(t)-v(t)\right\| d t \leq K_{1}\left(\int_{0}^{T}\left\|v^{N}(t)-v(t)\right\|^{2} d t\right)^{\frac{1}{2}}
$$

and using the strong convergence in $L_{2}\left(0, T, \mathcal{H}_{0}(\Omega)\right)$ we obtain

$$
\lim _{N \rightarrow \infty}\left|\int_{0}^{T} \tilde{S}_{1}^{N}(t) d t\right|=0
$$

$s_{2}^{N}(t)$ : Due to $v \in L_{2}\left(0, T, \mathcal{H}_{1}(\Omega)\right)$ according to Lemma 8.6 it follows from the estimate

$$
\begin{aligned}
\left|\int_{0}^{T} s_{2}^{N}(t) d t\right| & \leq \frac{1}{2} \cdot \int_{0}^{T}\left\|v^{N}(t)-v(t)\right\|\|\nabla a\|_{\infty}\|v(t)\| d t \\
& \leq K_{2}\left(\int_{0}^{T}\left\|v^{N}(t)-v(t)\right\|^{2} d t\right)^{\frac{1}{2}}
\end{aligned}
$$

the same behavior as above for $\tilde{S}_{1}^{N}(t)$ :

$$
\lim _{N \rightarrow \infty}\left|\int_{0}^{T} s_{2}^{N}(t) d t\right|=0 .
$$


$s_{1}^{N}(t)$ : For every $t \in[0, T]$ the function $v(t)$ is the weak limit of $v^{N}(t)$ in $\mathcal{H}_{0}(\Omega)$ (see Lemma 8.6). Hence also the weak limit $v(t)$ satisfies the estimate (8.7), and we find

$$
\begin{aligned}
\left|\int_{0}^{T} s_{1}^{N}(t) d t\right| & =\left|\sum_{k=0}^{N-1} \int_{t_{k}}^{t_{k+1}} s_{1}^{N}(t) d t\right| \\
& \leq \frac{1}{2 \varepsilon} \sum_{k=0}^{N-1} \int_{t_{k}}^{t_{k+1}} \int_{t_{k-1}}^{t_{k}}\left\|v^{N}(\tau)-v^{N}(t)\right\|\|\nabla a\|_{\infty}\|v(t)\| d \tau d t \\
& \leq \frac{K_{3}}{\varepsilon} \sum_{k=0}^{N-1} \int_{t_{k}}^{t_{k+1}} \int_{t_{k-1}}^{t_{k}}\left\|v^{N}(\tau)-v^{N}(t)\right\| d \tau d t
\end{aligned}
$$

Here we have $\tau \leq 0$ if $k=0$. This summand is estimated separately: Due to (8.7) and (8.8) we find

$$
\frac{K_{3}}{\varepsilon} \int_{0}^{\varepsilon} \int_{-\varepsilon}^{0}\left\|v^{N}(\tau)-v^{N}(t)\right\| d \tau d t \leq \frac{K_{3}}{\varepsilon} \int_{0}^{\varepsilon} \int_{-\varepsilon}^{0}\left\{\left\|v^{N}(\tau)\right\|+\left\|v^{N}(t)\right\|\right\} d \tau d t \leq \frac{K_{4}}{N}
$$

The rest of the above sum can be treated with the inequality of Friedrichs (see [11]): Let $\delta>0$ be fixed. Then there is some number $M_{\delta} \in \mathbb{N}$ with

$$
\begin{aligned}
\left\|v^{N}(\tau)-v^{N}(t)\right\| & \leq \sum_{j=1}^{M_{\delta}}\left|\left(v^{N}(\tau)-v^{N}(t), a_{j}\right)\right|+\delta\left\{\left\|\nabla v^{n}(\tau)\right\|+\left\|\nabla v^{n}(t)\right\|\right\} \\
& =: \quad g_{1}^{N}(\tau, t)+\delta g_{2}^{N}(\tau, t) .
\end{aligned}
$$

In the first term $g_{1}^{N}$ we have $\tau, t \geq 0$. Using (8.15) it follows

$$
\left|\left(v^{N}(\tau)-v^{N}(t), a_{j}\right)\right| \leq \bar{K}_{j}|\tau-t| \leq 2 \bar{K}_{j} \varepsilon
$$

and we obtain for the integral over $g_{1}^{N}$ the estimate

$$
\frac{K_{5}}{\varepsilon}\left(\sum_{j=1}^{M_{\delta}} \bar{K}_{j}\right) N \varepsilon^{3}=K_{5}\left(\sum_{j=1}^{M_{\delta}} \bar{K}_{j}\right) \frac{T^{2}}{N}
$$

Thus it remains to show that the integral over $g_{2}^{N}$ remains bounded as $N \rightarrow \infty$.

Due to

$$
\frac{K_{3}}{\varepsilon} \sum_{k=1}^{N-1} \int_{t_{k}}^{t_{k+1}} \int_{t_{k-1}}^{t_{k}}\left\{\left\|\nabla v^{N}(\tau)\right\|+\left\|\nabla v^{N}(t)\right\|\right\} d \tau d t
$$




$$
\begin{aligned}
& \leq K_{3} \sum_{k=1}^{N-1}\left\{\int_{t_{k}}^{t_{k+1}}\left\|\nabla v^{N}(t)\right\| d t+\int_{t_{k-1}}^{t_{k}}\left\|\nabla v^{N}(\tau)\right\| d \tau\right\} \\
& \leq 2 K_{3} \int_{0}^{T}\left\|\nabla v^{N}(t)\right\| d t \\
& \leq K_{6}\left(\int_{0}^{T}\left\|\nabla v^{N}(t)\right\|^{2} d t\right)^{\frac{1}{2}} \\
& \leq K_{7}
\end{aligned}
$$

according to (8.7), also the limit procedure

$$
\lim _{n \rightarrow \infty}\left|\int_{0}^{T} s_{1}^{N}(t) d t\right|=0
$$

holds true.

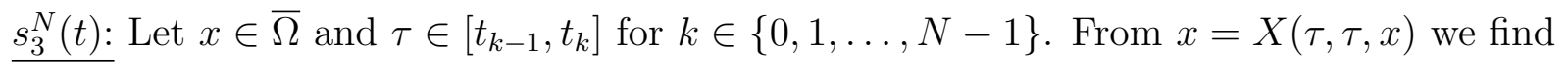

$$
v(t, x)-v\left(t, X\left(t_{k-1}, \tau, x\right)\right)=\int_{t_{k-1}}^{\tau}\left[v^{N}(\sigma) \cdot \nabla v(t)\right] \circ X(\sigma, \tau, x) d \sigma .
$$

From the Hölder inequality and the Sobolev imbedding theorem it follows

$$
\begin{aligned}
\left|s_{3}^{N}(t)\right| & \leq \frac{t-t_{k}}{2 \varepsilon^{2}}\left|\int_{t_{k-1}}^{t_{k}} \int_{t_{k-1}}^{\tau}\left(v(t) \cdot \nabla a,\left[v^{N}(\sigma) \cdot \nabla v(t)\right] \circ X(\sigma, \tau, \cdot)\right) d \sigma d \tau\right| \\
& \leq \frac{1}{2 \varepsilon} \int_{t_{k-1}}^{t_{k}} \int_{t_{k-1}}^{\tau}\|v(t)\|_{0,6}\|\nabla a\|_{\infty}\left\|v^{N}(\sigma)\right\|_{0,3}\|\nabla v(t)\| d \sigma d \tau \\
& \leq K_{7}\|\nabla v(t)\|^{2} \cdot \int_{t_{k-1}}^{t_{k}}\left\|\nabla v^{N}(\sigma)\right\| d \sigma \\
& \leq K_{z} \sqrt{\varepsilon}\|\nabla v(t)\|^{2}\left(\int_{-T}^{+T}\left\|\nabla v^{N}(\sigma)\right\|^{2} d \sigma\right)^{\frac{1}{2}} \\
& \leq K_{8} \sqrt{\varepsilon}\|\nabla v(t)\|^{2} .
\end{aligned}
$$


Here the last estimate is due to (8.7) and (8.9), and using (8.15) also the last limit transition is proved:

$$
\lim _{N \rightarrow \infty}\left|\int_{0}^{T} s_{3}^{N}(t) d t\right| \leq \lim _{N \rightarrow \infty} \frac{K_{g}}{\sqrt{N}} \cdot \int_{0}^{T}\|\nabla v(t)\|^{2} d t=0 .
$$

After these preparations we are now ready to prove that the function $v$ from Lemma 8.6 satisfies the assertions of Theorem 8.3:

Proof of Theorem 8.3: Using (8.6) and the compactness of the imbedding

$$
\mathcal{H}_{1}(\Omega) \hookrightarrow \mathcal{H}_{0}(\Omega)
$$

we find without any restriction of generality that the subsequence $\left(v_{0}^{N_{k}}\right)_{k}$ of the initial values of the solutions $v^{N_{k}}$ from Lemma 8.6 converges to some function $v_{0} \in \mathcal{H}_{0}(\Omega)$ strongly in $\mathcal{H}_{0}(\Omega)$. From here we quote with the weak convergence according to Lemma 8.6 that the limit function $v$ satisfies the energy inequality: For all $t \in[0, T]$ we have

$$
\begin{aligned}
\|v(t)\|^{2}+2 \nu \int_{0}^{t}\|\nabla v(\tau)\|^{2} d \tau & \leq \liminf _{k \rightarrow \infty}\left\|v^{N_{k}}(t)\right\|^{2}+2 \nu \liminf _{k \rightarrow \infty} \int_{0}^{t}\left\|\nabla v^{N_{k}}(\tau)\right\|^{2} d \tau \\
& \leq \lim _{k \rightarrow \infty}\left\|v_{0}^{N_{k}}-v_{0}+v_{0}\right\|^{2}=\left\|v_{0}\right\|^{2}
\end{aligned}
$$

Together with the weak continuity of the function $t \rightarrow v(t)$ in $\mathcal{H}_{0}(\Omega)$ at time $t=0$ it follows the strong continuity of this function at time $t=0$ as in (5.28), (5.29), hence (8.2) holds true. It remains to prove (8.3), where we can restrict our considerations to test functions of the form $\phi_{i}=\varphi \cdot a_{i}$ with $\varphi \in C_{0}^{\infty}((0, T))$ and $a_{i} \in \mathcal{V} \subset C_{0, \sigma}^{\infty}(\Omega)$, using again a density argument. To do so, we multiply the first equation of the problem $\left(N_{\varepsilon_{N_{k}}}\right)$ by $\varphi_{i}$, integrate over $\Omega_{T}$ and obtain with help of partial integration

$$
\int_{0}^{T}\left\{\left(v^{N_{k}(t), a_{i}}\right) \varphi^{\prime}(t)-\nu\left(\nabla v^{N_{k}}(t), \nabla a_{i}\right) \varphi(t)\right\} d t=-\int_{0}^{T}\left(v^{N_{k}}(t) \cdot \nabla a_{i}, v^{N_{k}}(t)\right) \varphi(t) d t .
$$

Finally, using the weak convergence of the subsequence in $L_{2}\left(0, T, \mathcal{H}_{0}(\Omega)\right)$ as well as in $L_{2}\left(0, T, \mathcal{H}_{1}(\Omega)\right)$ to the limit function $v$, respectively, and Theorem 8.7 we obtain (8.3) as $k \rightarrow \infty$, and the theorem is proved.

\section{Local strong convergence}

Let $T>0, N \in \mathbb{N}(N \geq 2), \varepsilon_{N}:=\frac{T}{N}$, and let $v^{N}$ denote the solution of the problem $\left(N_{\varepsilon_{N}}\right)$ with initial value $v_{0}^{N} \in H_{m}(\Omega) \cap \mathcal{H}_{1}(\Omega)$ for $m \in\{3,4\}$. According to Corollary 
7.2 , the function $v^{N}$ coincides with the solution of the problem $\left(\tilde{N}_{\varepsilon_{N}}\right)$ with initial value $f \in H_{m-2}(\Omega) \cap \mathcal{H}_{1}(\Omega)$.

In the last section we have shown that the sequences $\left(v^{N}\right)_{N}$ and $\left(v_{0}^{N}\right)_{N}$ have accumulation points $v$ and $v_{0}$, respectively, such that $v$ is a weak solution of the problem $\left(N_{0}\right)$ with initial value $v_{0} \in \mathcal{H}_{0}(\Omega)$.

The next theorem describes local properties of such a pair of accumulation points.

Theorem 9.1. Let $T>0, N \in \mathbb{N}(N \geq 2)$ and $\varepsilon_{N}:=\frac{T}{N}$. Let the assumptions of Theorem 8.3 be satisfied, and let $f \in H_{m-2}(\Omega) \cap \mathcal{H}_{1}(\Omega)$ for $m \in\{3,4\}$ denote the initial value of the problem $\left(\tilde{N}_{\varepsilon_{N}}\right)$.

Then there exists some number $T^{*}=T^{*}(\Omega, \nu, f)$ with $0<T^{*} \leq T$ such that the following statements hold true: Every problem $\left(N_{0}\right)$ from Theorem 8.3 is uniquely solvable in $\left[0, T^{*}\right]$, and for its solution $v$ we find

$$
v \in C\left(\left[0, T^{*}\right], H_{m}(\Omega) \cap \mathcal{H}_{1}(\Omega)\right), \quad \partial_{t} v \in C\left(\left[0, T^{*}\right], H_{m-2}(\Omega) \cap \mathcal{H}_{1}(\Omega)\right),
$$

and for $m=4$ even $\partial_{t}^{2} v \in C\left(\left[0, T^{*}\right], \mathcal{H}_{0}(\Omega)\right)$. Moreover we have

$$
\partial_{t} v(0)=f .
$$

To prove this theorem we consider first in $\Omega$ the stationary nonlinear Navier-Stokes boundary value problem

$$
\nu P \Delta u_{0}-P\left(u_{0} \cdot \nabla u_{0}\right)=f
$$

and show in the next lemma that every function $v_{0}$ from Theorem 8.3 is a weak solution of this problem. To do so let us recall:

Definition 9.2. A function $u_{0} \in \mathcal{H}_{1}(\Omega)$ is a weak solution of the Navier-Stokes problem (9.2), if for all test functions $\varphi \in C_{0, \sigma}^{\infty}(\Omega)$ the identity

$$
-\nu\left(\nabla u_{0}, \nabla \varphi\right)+\left(u_{0} \cdot \nabla \varphi, u_{0}\right)=(f, \varphi)
$$

is valid.

Lemma 9.3. Let $f \in H_{m-2}(\Omega) \cap \mathcal{H}_{1}(\Omega)$. Then every function $v_{0}$ from Theorem 8.3 is a weak solution of the problem (9.2) according to the definition 9.2, and we find $v_{0} \in$ $H_{m}(\Omega) \cap \mathcal{H}_{1}(\Omega)$.

Proof: For simplification let us assume the convergence of the whole sequence $\left(v_{0}^{N}\right)_{N}$ from Therorem 8.3 to the limit function $v_{0}$. From (8.6) we know that this convergence is weak with respect to $\mathcal{H}_{1}(\Omega)$ and strong with respect to $\mathcal{H}_{0}(\Omega)$, and that $v_{0} \in \mathcal{H}_{1}(\Omega)$ is true. Now let $\mathcal{V}:=\left\{a_{i} \mid i \in \mathbb{N}\right\}$ be a complete orthonormal system in $\mathcal{H}_{1}(\Omega)$. Then we can choose $\mathcal{V} \subset C_{0, \sigma}^{\infty}(\Omega)$ and restrict us in (9.3) for $\varphi$ on basis functions of the type $a=a_{i}$. Due to the 
weak convergence in $\mathcal{H}_{1}(\Omega)$ the first part of the lemma is proved, if, using (8.13), we can show

$$
\lim _{N \rightarrow \infty}\left\{\frac{1}{2 \varepsilon}\left(a \circ X_{-1}-a \circ X_{-1}^{-1}, v_{0}^{N}\right)-\left(v_{0} \cdot \nabla a, v_{0}\right)\right\}=0
$$

According to Section 7 here the measure conserving mapping $X_{-1}:=X_{-\varepsilon,-2 \varepsilon}$ is constructed from the velocity field $\bar{v}^{N}=\tilde{v}^{N-1}$, and we obtain as in the proof of Lemma 8.5 for every $x \in \bar{\Omega}$ the identity

$$
a \circ X_{-1}(x)-a \circ X_{-1}^{-1}(x)=\int_{-2 \varepsilon}^{-\varepsilon}\left\{\left[\tilde{v}^{N-1}(\tau) \cdot \nabla a\right] \circ X(\tau,-2 \varepsilon, x)+\left[\tilde{v}^{n-1}(\tau) \cdot \nabla a\right] \circ X(\tau,-\varepsilon, x)\right\} d \tau .
$$

The assertion(9.4) then follows by a suitable decomposition as in Theorem 8.7.

For the asserted regularity statement, using the estimate of Cattabriga for the equation

$$
P \Delta v_{0}=\frac{1}{\nu}\left\{f+P\left(v_{0} \cdot \nabla v_{0}\right)\right\}
$$

we have to prove $v_{0} \cdot \nabla v_{0} \in H_{m-2}(\Omega)$. This can be done with help of the Sobolev imbedding theorem and a suitable bootstrapping procedure as in $([14])$ : Due to $v_{0} \in \mathcal{H}_{1}(\Omega)$ we have $v_{0} \in L_{6}(\Omega)$, and using

$$
\left\|v_{0} \cdot \nabla v_{0}\right\|_{0, \frac{3}{2}} \leq\left\|\nabla v_{0}\right\| \cdot\left\|v_{0}\right\|_{0,6}
$$

it follows $v_{0} \cdot \nabla v_{0} \in L_{\frac{3}{2}}(\Omega)$, hence $v_{0} \in H_{2, \frac{3}{2}}(\Omega)$ and thus $v_{0} \in L_{\infty}(\Omega)$. Since $\left\|v_{0} \cdot \nabla v_{0}\right\| \leq$ $\left\|v_{0}\right\|_{\infty} \cdot\left\|\nabla v_{0}\right\|$ it follows $v_{0} \cdot \nabla v_{0} \in L_{2}(\Omega)$, hence $v_{0} \in H_{2}(\Omega)$.

From

$$
\left\|\nabla\left(v_{0} \cdot \nabla v_{0}\right)\right\|=\left\|\nabla v_{0}\right\|_{0,4}^{2}+\left\|v_{0}\right\|_{\infty}\left\|\nabla^{2} v_{0}\right\| \leq c_{\Omega}\left\|v_{0}\right\|_{2,2}^{2}
$$

we quote $v_{0} \cdot \nabla v_{0} \in H_{1}(\Omega)$, hence $v_{0} \in H_{3}(\Omega)$ and thus $\nabla v_{0} \in L_{\infty}(\Omega)$. Using

$$
\left\|\nabla^{2}\left(v_{0} \cdot \nabla v_{0}\right)\right\| \leq 3\left\|\nabla v_{0}\right\|_{\infty}\left\|\nabla^{2} v_{0}\right\|+\left\|v_{0}\right\|\left\|v_{0}\right\|_{3,2} \leq c_{\Omega}\left\|v_{0}\right\|_{3,2}^{2}
$$

we finally obtain $v_{0} \cdot \nabla v_{0} \in H_{m-2}(\Omega)$.

From (9.5) it follows that the first statement of Lemma 9.3 and thus the assertion (9.1) from Theorem 9.1 does not necessarily hold true, if the same function $\bar{v}$, which is needed for the construction of the initial data of the problem $\left(N_{\varepsilon_{N}}\right)$, is used for all $N \in \mathbb{N}$.

Proof of Theorem 9.1: For every weakly solvable problem from Theorem 8.3, due to the regularity of its initial value $v_{0}$, there exists a number $\tilde{T}=\tilde{T}\left(\Omega, \nu, v_{0}\right)$ with $0<\tilde{T} \leq T$ such that this problem can be solved uniquely in $[0, \tilde{T}]$, and that its strong solution, due to the validity of the compatibility condition at time $t=0$, has all the properties asserted in 9.1 (compare also [14]). Since the norms of the initial data can be estimated by the norm of $f$ there is a unique existence interval $\left[0, T^{*}\right]$ of positive length.

Thus the solution of problem $\left(N_{0}\right)$ from Theorem 9.1 is a solution of the following NavierStokes initial boundary value problem $\left(\tilde{N}_{0}\right)$ in $\left[0, T^{*}\right]$ : 
Let $T>0$. Construct a velocity field $v=\left(v_{1}, v_{2}, v_{3}\right)$ and some pressure function $p$ as a solution of the system

$$
\begin{aligned}
\partial_{t} v-\nu \Delta v+\nabla p & =-v \cdot \nabla v \\
\nabla \cdot v & =0 \\
v_{\mid \partial \Omega} & =0 \\
\partial_{t} v_{\mid t=0} & =f .
\end{aligned}
$$$$
\left(\tilde{N}_{0}\right)
$$

Since the stationary problem (9.2) can be solved uniquely for sufficiently large $\nu$ or sufficiently small $f$ (see [13]) we find that in this case the problem $\left(\tilde{N}_{0}\right)$ is locally, i.e. in $\left[0, T^{*}\right]$, uniquely solvable and the whole sequence $\left(v_{0}^{N}\right)_{N}$ of the initial values of the problems $\left(N_{\varepsilon_{N}}\right)$ from Theorem 8.3 converges to the uniquely determined initial value $v_{0}$.

\section{References}

[1] Adams, A., Fournier, J.J.F.: Sobelev spaces. Academic Press Oxford (2003).

[2] Cattabriga, L.: Su un problema al contorno relativo al sistema die equazioni di Stokes. Rend. Sem. Math. Univ. Padova 31 (1960), 308-340.

[3] Heywood, J. G.: The Navier-Stokes equations, on the existence, regularity and decay of solutions. Ind. Univ. Mat. Journal 29 No. 5 (1980), 639-681.

[4] Heywood, J. G., R. Rannacher: Finite element approximation of the nonstationary Navier-Stokes problem I, regularity of solutions and second-order error estimates for spatial discretisation. Siam J. Numer. Anal. 19 No. 2 (1982), 275-311.

[5] Hopf, E.: Über die Anfangswertaufgabe für die hydrodynamischen Grundgleichungen. Math. Nachr. 4 (1951), 213-231.

[6] Kiselev, A. A., O. A. Ladyzhenskaya: On the existence and uniqueness of the solution of the nonstationary problem for a viscous incompressible fluid. Izv. Akad. Nauk SSSR Ser. Mat. 21 (1957), 655-680.

[7] Ladyzhenskaya, O. A.: The mathematical theorie of viscous incompressible flow. Gordon and Breach New York (1969).

[8] Prodi, G.: Teoremi di tipo locale per il sistema di Navier-Stokes et stabilità delle soluzione stazionarie. Rend. Sem. Math. Univ. Padova 32 (1962), 374-397.

[9] Rautmann, R.: On optimum regularity of Navier-Stokes solutions at time $t=0$. Math. Z. 184 (1983), 141-149. 
[10] Serrin, J.: The initial value problem for the Navier-Stokes equations. Nonlinear Problems, Proceedings of a Symposium (Madison 1962), Univ. of Wisconsin Press (1963), 69-98.

[11] Shinbrot, M.: Lectures on fluid mechanics. Gordon and Breach New York (1973).

[12] Sohr, H.: The Navier-Stokes Equations. Birkhäuser Basel (2001).

[13] Temam, R.; Navier-Stokes Equations. AMS Chelsea, New York (2001).

[14] Temam, R.: Behaviour at time $t=0$ of the solutions of semi-linear evolution equations. MRC Technical Summary Report 2162 Madison, Univ. of Wisconsin (1980).

[15] Walter, W.: Differential and integral inequalities. Springer Berlin-Heidelberg-New York (1970).

[16] Walter, W.: Gewöhnliche Differentialgleichungen. Springer Berlin-Heidelberg-New York (2000). 